\title{
A Location-Inventory-Routing Problem in Forward and Reverse Logistics Network Design
}

\author{
Qunli Yuchi, ${ }^{1,2}$ Zhengwen He, $^{1,2}$ Zhen Yang, $^{1,2}$ and Nengmin Wang \\ ${ }^{1}$ School of Management, Xian Jiaotong University, No. 28 Xianning Road, Xi'an, Shaanxi 710049, China \\ ${ }^{2}$ The Key Lab of the Ministry of Education for Process Control \& Efficiency Engineering, No. 28 Xianning Road, Xian, \\ Shaanxi 710049, China
}

Correspondence should be addressed to Nengmin Wang; nengmin.wang@163.com

Received 1 February 2016; Revised 25 May 2016; Accepted 6 June 2016

Academic Editor: Gabriella Bretti

Copyright (C) 2016 Qunli Yuchi et al. This is an open access article distributed under the Creative Commons Attribution License, which permits unrestricted use, distribution, and reproduction in any medium, provided the original work is properly cited.

\begin{abstract}
We study a new problem of location-inventory-routing in forward and reverse logistic (LIRP-FRL) network design, which simultaneously integrates the location decisions of distribution centers (DCs), the inventory policies of opened DCs, and the vehicle routing decision in serving customers, in which new goods are produced and damaged goods are repaired by a manufacturer and then returned to the market to satisfy customers' demands as new ones. Our objective is to minimize the total costs of manufacturing and remanufacturing goods, building DCs, shipping goods (new or recovered) between the manufacturer and opened DCs, and distributing new or recovered goods to customers and ordering and storage costs of goods. A nonlinear integer programming model is proposed to formulate the LIRP-FRL. A new tabu search (NTS) algorithm is developed to achieve near optimal solution of the problem. Numerical experiments on the benchmark instances of a simplified version of the LIRP-FRL, the capacitated location routing problem, and the randomly generated LIRP-FRL instances demonstrate the effectiveness and efficiency of the proposed NTS algorithm in problem resolution.
\end{abstract}

\section{Introduction}

The need for designing distribution network to achieve a variety of the supply chain objects with the overall productivity has received considerable attentions and becomes more and more stronger in recent years. As Javid and Azad [1] said, location allocation problem, inventory control problem, and the vehicle routing problem are the most considered subproblems in designing a distribution network. Liu and Lee [2] showed that the routing and inventory decisions affect the location decision. Also, Viswanathan and Mathur [3] found that transportation costs will increase when the order quantity during each production runs decreases. Similar conclusions have also been found by Zhang et al. [4]; they pointed out that the above three subproblems are strongly correlated and mutually affect each other. Considering the three subproblems at the same time may provide a comprehensive estimation in constructing an efficient productiondistribution network. This gives rise to the researches on the location-inventory-routing problem (LIRP).
Differing in inventory policies used, the types of facilities located, and the vehicle routing strategies adopted, lots of researches on LIRP in forward logistics network design have been reported. Liu and Lee [2] studied a LIRP using order up to level inventory policy. A route-first and location-allocation second heuristic method was proposed by them to solve LIRP. Ambrosino and Scutella [5] studied a LIRP with the consideration of customers service level. Using $(Q, R)$ inventory policy, Shen and Qi [6] proposed a Lagrangian relaxation and a rank and search algorithm to solve the LIRP. Following the researches of [6], Javid and Azad [1] solved the same problem by using a tabu search and simulated annealing. Guerrero et al. [7] proposed a column generation, Lagrangian relaxation, and local search combined heuristic for a multiperiod LIRP.

As Ravi et al. [8] said, the forces of economic factors, legislation, corporate citizenship, and environmental protection problems are driving the increasing interests and investments of enterprises in reverse logistics. These motivate researchers to develop optimization models for reverse supply network design in the past decades (Vahdani and Naderi-Beni [9]). 
Chen et al. [10] concluded that the consideration of reverse logistics in distribution network design can greatly reduce the logistics costs without interrupting the forward flows. Taking both the forward logistics and reverse logistics into account, many researches on facility location, vehicle routing, and inventory management had been presented.

Vahdani et al. [11] focused on a fuzzy facility locationallocation problem in closed loop logistics. Differing from [11], Vahdani et al. [12,13] proposed a fuzzy possibility programming method to solve multiobjective facility problems in forward and reverse logistic.

Under the background of forward and reverse logistic, different inventory models had also been developed in the literature. Richter [14] proposed the first inventory model in a closed logistic using EOQ model. It assumed that customers' demands can be satisfied by newly made products or by repaired products. For a given fixed waste disposal rate, the optimal minimum cost expression was derived. Shi et al. [15] considered an inventory policy under uncertain customer demand. A Lagrangian relaxation based approach was proposed.

Vehicle routing decision is another important research subject in forward and reverse logistic management. Zachariadis and Kiranoudis [16] studied a VRP problem under the background of forward and reverse logistic. A tabu search method was proposed. For a similar VRP problem, Goksal et al. [17] developed a particle swarm optimization (PSO) based approach for its resolution, in which a variable neighborhood descent (VND) algorithm was used as a local search to explore solution space.

Different combination of facility location decision, inventory management decision, and vehicle routing decision gives rise to different research problems in forward and reverse logistics. For example, Liu et al. [18] proposed a hybrid particle swarm optimization to solve a location routing problem using grey systems theory in reverse logistics. Yu and Lin [19] presented a location routing problem with simultaneous pickup and delivery, which was solved via a multistart simulated annealing algorithm with multistart hill climbing. Modeling the problem as a bilevel programming problem, Wang et al. [20] studied realistic location-inventory problem in reverse logistic of a China B2C company. Li et al. [21] solved a location and inventory control problem by a two-stage heuristic algorithm that combined Lagrangian relaxation method with an ant colony algorithm.

Due to the intrinsic complexity of the problem in mathematics, to our best knowledge, only a few researches, except that of Li et al. [22], simultaneously considered the decisions of the facility location, the inventory management, and vehicle routing in forward and reverse logistics in the literature. In their model, they consider retrieving those products that customers dislike to the DCs. As these products are new or unused, they can be sold to customers directly with no need to be returned to manufacturer for repair.

However, as the work of Adler et al. [23] indicated, used (old or damaged) products (e.g., the engines of Caterpillar Company) can also be retrieved, recovered, and returned to the market to satisfy customers' demands as new ones.
This realistic new feature motivates us to focus on goods production, distribution, and recycling.

A location-inventory-routing problem in forward and reverse logistic (LIRP-FRL) network design is studied in this paper. The LIRP-FRL simultaneously integrates the location decisions of distribution centers (DCs), the inventory policies of opened DCs, and the vehicle routing decision in serving customers, in which new goods are produced and damaged goods are repaired by a manufacturer and then transported to opened DCs. Vehicles that start from and end in the same DC distribute new or recovered goods to satisfy the demands of customers and retrieve damaged goods. Our objective is to minimize the total costs of manufacturing and remanufacturing goods, building DCs, shipping goods (new or recovered) between the manufacturer and opened DCs, distributing new or recovered goods to customers, and retrieving damaged goods from customers to DCs and the inventory costs of DCs including ordering new or recovered goods and holding these goods.

Without taking the inventory strategy of DCs into account, our problem is simplified to the classical capacitated location routing problem (CLRP) [24], which is NP-hard in strong sense. Consequently, our problem is also NPhard. Further, the LIRP-FRL is a nonlinear problem as the EOQ inventory policy is adopted. Due to its complexity, we focus on finding near optimal solutions for the LIRPFRL. A new tabu search (NTS) algorithm is proposed to find approximately optimal solutions. This algorithm probabilistically accepts a second best solution to change search direction to achieve this goal. Numerical experiments of the CLRP benchmarks demonstrate the effectiveness and the efficiency of the proposed NTS method in obtaining high quality solutions in a very reasonable time. Computational and sensitivity analysis results on randomly generated LIRPFRL instances are also reported.

The rest of this paper is organized as follows. In Section 2, a mathematical model is developed to describe the studied LIRP-FRL. A tabu search that seeks to search near optimal solution for the LIRP-FRL is proposed in Section 3. Algorithm performance evaluations are conducted in Section 4. Finally, we conclude the paper in Section 5.

\section{Mathematical Model}

Given a set of potential sites of distribution centers (DCs), the studied location-inventory-routing problem in forward and reverse logistic (LIRP-FRL) network design is to optimally determine the locations of DCs and their order quantities from a manufacturer, the product flows (forward and reverse) between the manufacturer and the opened DCs, and the paths of vehicles in serving customers. We assume that each route starts from and ends in the same DC, by which new or recovered products are delivered to satisfy the demands of customers for each cycle, and damaged products are retrieved. The objective of the LIRP-FRL is to minimize the sum of the opening costs and the inventory costs of DCs, the setup costs of vehicles, and the transportation costs of (new, repaired, and damaged) products. An example of the studied LIRP-FRL is illustrated in Figure 1. 


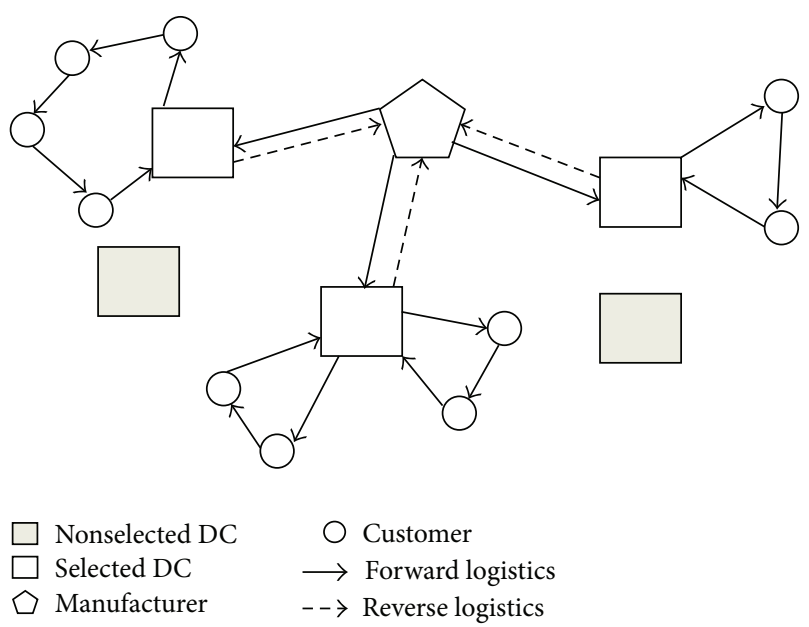

FIGURE 1: Location-inventory-routing problem in forward and reverse logistic.

2.1. Notations. The notations are described as follows:

I: the index set of customers,

$J$ : the index set of potential distribution centers,

$K$ : the index set of vehicles,

$S$ : the merged set of customers and potential distribution centers $(I \cup J)$,

$V_{j}$ : the maximum capacity of the distribution center $j, j \in J$,

$\mathrm{FC}_{j}$ : the fixed opening cost of establishing distribution center $j, j \in J$,

$Q$ : the maximum capacity of homogeneous vehicle,

$c_{i j}$ : the transportation cost of a vehicle on $\operatorname{arc}(i, j), i$, $j \in S, i \neq j$,

$f_{j}$ : the setup cost of using a vehicle at distribution center $j, j \in J$,

$c^{j}$ : the product unit transportation cost from the manufacturer to distribution center $j, j \in J$,

$A^{m}$ : the order cost of new products of DCs per cycle,

$A^{r}$ : the order cost of recovered or repaired products of DCs per cycle,

$c^{m}$ : the cost of manufacturing a new product,

$c^{r}$ : the cost of repairing a damaged product to a new product,

$D_{i}$ : the demand of customers per cycle,

$h_{j}$ : the unit holding cost of distribution center $j, j \in J$, $r$ : the rate of return in percentage,

$c^{d}$ : the unit cost of disposal of useless retrieved products,

$u$ : the percentage of reuse of retrieved products.
2.2. Decision Variables. The decision variables are described as follows:

$Q_{j}^{m}$ is the order quantity of new products of DC $j \in J$ for each period,

$Q_{j}^{r}$ is the order quantity of recovered products of DC $j \in J$ for each period,

$M_{j}$ is the order times of new products from DC $j \in J$ during each period,

$R_{j}$ is the order times of recovered products from DC $j \in J$ during each period,

$x_{i j k}=\{1$, if $\operatorname{arc}(i, j)$ is travelled by vehicle $k ; 0$, otherwise $(i, j \in S, k \in K)$,

$y_{i j}=\{1$, if customer $i \in I$ is served by distribution center $j \in J ; 0$, otherwise $\}$,

$Z_{j}=\{1$, if distribution center $j \in J$ is established; 0 , otherwise\}.

2.3. Cost Analysis. To simplify the presentation, we first abbreviate the costs related to the LIRP-FRL as follows:

(1) The total fixed cost of opening distribution centers is

$$
\operatorname{FIXCOST}=\sum_{j \in J} \mathrm{FC}_{j} \cdot z_{j} .
$$

(2) The total cost of ordering new and recovered products is

$\mathrm{OC}=\sum_{j \in J} A^{m} \cdot \frac{\sum_{i \in I} D_{i}(1-u) y_{i j}}{Q_{j}^{m}}+A^{r} \cdot \frac{\sum_{i \in I} D_{i} u y_{i j}}{Q_{j}^{r}}$.

(3) The total cost of producing new products and recovering retrieved products is

$$
\mathrm{MR}=\sum_{j \in J} \sum_{i \in I} D_{i} \cdot\left((1-u) \cdot c^{m}+u \cdot c^{r}\right) \cdot y_{i j} \cdot
$$

(4) The total cost of disposal of useless retrieved products is

$$
\mathrm{DISP}=\sum_{j \in J} \sum_{i \in I} D_{i} \cdot(r-u) \cdot c^{d} \cdot y_{i j} .
$$

(5) The transportation cost between the manufacturer and distribution centers is

$$
\text { TRAN }=\sum_{j \in J} c^{j} \sum_{i \in I} D_{i} \cdot(1+u) \cdot y_{i j}
$$

(6) The transportation cost between distribution centers and customers is

$$
\mathrm{TRC}=\sum_{k \in K} \sum_{j \in S} \sum_{i \in S} f_{j} \cdot x_{i j k}+\sum_{k \in K} \sum_{j \in S} \sum_{i \in I} c_{i j} \cdot x_{i j k} .
$$


Given the locations of DCs and the routes of vehicles, the LIRP-FRL is reduced to the inventory model of Teunter [25]. Some of their valuable conclusions can be directly applied to our LIRP-FRL.

Proposition 1 (Teunter [25]). "It is reasonable to restrict our attention to those policies with either $M=1$ or $R=1$."

Teunter [25] concluded that the policies with either $M=$ 1 or $R=1$ can obtain near optimal solution with less computational time by comparing with that of achieving optimal solution. Since our problem is more complicated than that of Teunter [25], to simplify the resolution of LIRPFRL, we consider only the two cases in our implementation. The two cases are as follows.

Case $1(1, M)$. That means $R=1$; in this case the order times of recovered products for each DC are equal to one.

Case $2(R, 1)$. That means $M=1$; in this case the order times of new products for each DC are equal to one.

For Case $1(1, M)$, the holding cost is

$$
\mathrm{HM}=\sum_{j \in J} \frac{1}{2} h_{j}\left((1-u) \cdot Q_{j}^{m}+u \cdot Q_{j}^{r}+Q_{j}^{r} \cdot \frac{u}{r}\right) \cdot z_{j} .
$$

Consequently, the total cost is denoted as

$$
\begin{aligned}
\mathrm{TCM}= & \text { FIXCOST }+\mathrm{OC}+\mathrm{MR}+\mathrm{DISP}+\mathrm{TRAN} \\
& +\mathrm{TRC}+\mathrm{HM} .
\end{aligned}
$$

For Case $2(R, 1)$, the holding cost is

$$
\begin{aligned}
\mathrm{HR} & =\sum_{j \in J} \frac{1}{2} h_{j}\left((1-u) \cdot Q_{j}^{m}+u \cdot Q_{j}^{r}\right. \\
& \left.+\left(r\left(Q_{j}^{m}+Q_{j}^{r}\right)-(r-u) \cdot\left(Q_{j}^{m}+\frac{u}{1-u} Q_{j}^{m}\right)\right) \frac{u}{r}\right) \\
& \cdot z_{j} .
\end{aligned}
$$

The corresponding total cost is

$$
\begin{aligned}
\mathrm{TCR}= & \text { FIXCOST }+\mathrm{OC}+\mathrm{MR}+\mathrm{DISP}+\mathrm{TRAN} \\
& +\mathrm{TRC}+\mathrm{HR} .
\end{aligned}
$$

2.4. Mathematical Model. The LIRP-FRL can be formulated as the following nonlinear mixed integer programming problem:

$$
\begin{array}{ll}
\min & \{\text { TCM, TCR }\}, \\
\text { s.t. } & \sum_{k \in K} \sum_{i \in S} x_{i j k}=1, \quad j \in I \\
& \sum_{j \in S} x_{i j k}-\sum_{j \in S} x_{j i k}=0, \quad i \in S, k \in K \\
& \sum_{j \in J} \sum_{i \in I} x_{i j k} \leq 1, \quad k \in K \\
& \sum_{j \in J} \sum_{i \in I} D_{i} x_{i j k} \leq Q, \quad k \in K
\end{array}
$$

$$
\begin{aligned}
& \sum_{i \in I} D_{i} y_{i j}-V_{j} \cdot z_{j} \leq 0, \quad j \in J \\
& y_{i j}+1 \geq \sum_{g \in I} x_{i g k}+\sum_{g \in S \backslash\{j\}} x_{g j k}, \\
& \qquad i \in J, j \in I, k \in K \\
& \sum_{j \in W} \sum_{i \in W} x_{i j k} \leq|W|-1, \quad W \subseteq I, k \in K \\
& x_{i j k} \in\{0,1\}, \quad i \in S, \quad j \in S, k \in K \\
& y_{i j} \in\{0,1\}, \quad i \in I, \quad j \in J \\
& z_{j} \in\{0,1\}, \quad j \in J .
\end{aligned}
$$

Constraints (12) state that each customer is exactly served by one vehicle. Constraints (13) are the equilibrium constraints of vehicle routes on nodes. Constraints (14) ensure that each vehicle can be used by at most one DC. Constraints (15) guarantee that the capacity of vehicle cannot be exceeded. Constraints (16) are the capacity constraints of distribution centers. Constraints (17) make sure that a customer can only be served by the vehicle which originates from the DC it is assigned to. Constraints (18) are the subtours elimination constraints of vehicle routes.

Proposition 2. For any distribution center, $j \in J$, its order quantities $Q_{j}^{m}$ and $Q_{j}^{r}$ can be represented as a function of customers' demands, respectively.

$$
\begin{aligned}
& \text { We have } Q_{j}^{m}=\sqrt{2 A^{m} \sum_{i \in I} D_{i} \cdot y_{i j} / h_{j}} \text { and } Q_{j}^{r}= \\
& \sqrt{2 A^{r} \cdot r \cdot \sum_{i \in I} D_{i} \cdot y_{i j} /(r+1) h_{j}} \text { for any } j \in J .
\end{aligned}
$$

Proof. It can be observed that, no matter case $(1, M)$ or case $(R, 1)$, decision variables $Q_{j}^{m}$ and $Q_{j}^{r}$ appear only in the objective function. For the case $(R, 1)$, the optimal value of $Q_{j}^{m}$ and $Q_{j}^{r}$ can be obtained by checking the extreme conditions: $\partial \mathrm{TCM} / \partial Q_{j}^{m}=0$ and $\partial \mathrm{TCM} / \partial Q_{j}^{r}=0$.

Since $\partial^{2} \mathrm{TCM} / \partial\left(Q_{j}^{m}\right)^{2}=\left(2 A^{m} \cdot(1-u) \sum_{i \in I} D_{i}\right.$. $\left.y_{i j}\right) /\left(Q_{j}^{m}\right)^{3} \geq 0$ and $\partial^{2} \mathrm{TCM} / \partial\left(Q_{j}^{r}\right)^{2}=\left(2 A^{r} \cdot u \sum_{i \in I} D_{i}\right.$. $\left.y_{i j}\right) /\left(Q_{j}^{r}\right)^{3} \geq 0$, consequently the extreme point is a minimum point. We obtain the optimal order quantities $Q_{j}^{m}=$ $\sqrt{\left(2 A^{m} \sum_{i \in I} D_{i} \cdot(1-u) y_{i j}\right) / h_{j}(1-((r-u) /(1-u)) \cdot(u / r))}$ and $Q_{j}^{r}=\sqrt{\left(A^{r} \cdot \sum_{i \in I} D_{i} \cdot y_{i j}\right) / h_{j}}$ and the corresponding order time $R_{j}=\left(u \cdot Q_{j}^{m}\right) /\left(1-u \cdot Q_{j}^{r}\right)$.

For the same reason, for the case $(1, M)$, we can derive that the order quantities $Q_{j}^{m}=\sqrt{\left(2 A^{m} \sum_{i \in I} D_{i} \cdot y_{i j}\right) / h_{j}}$ and $Q_{j}^{r}=\sqrt{\left(2 A^{r} \cdot r \cdot \sum_{i \in I} D_{i} \cdot y_{i j}\right) /(r+1) h_{j}}$ and the corresponding order time $M_{j}=\left(1-u \cdot Q_{j}^{r}\right) /\left(u \cdot Q_{j}^{m}\right)$.

Observe that, without considering the inventory strategy of these two cases, Cases 1 and 2, the LIRP-FRL is reduced to the classical capacitated location routing problem (CLRP), which is NP-hard in the strong sense. As a result, the LIRPFRL is also NP-hard. As Belenguer et al. [24] reported, to 


\begin{tabular}{|l|l|l|l|l|l|l|l|l|l|l|l|l|l|l|l|l|l|l|l|l|l|l|l|l|}
\hline 21 & 23 & 19 & 16 & 15 & 14 & 8 & 11 & 6 & 22 & 4 & 1 & 12 & 18 & 20 & 13 & 5 & 7 & 3 & 24 & 25 & 2 & 17 & 9 & 10 \\
\hline
\end{tabular}

FIGURE 2: An example of solution representation.

find CLRP optimal solution, even for the median size CLRP instances (with 50 customers and 5 potential distribution centers), significant research efforts need to be expensed. Thus, in this paper, we focus on finding near optimal solutions for the LIRP-FRL.

\section{New Tabu Search}

Using tabu list to avoid visited solutions to be revisited, the tabu search proposed by Glover [26] is one of the most effective approaches for solving mixed integer programming problems, which has been successfully applied to a variety of distribution network design problems. For the detailed introduction and application of the tabu search, we refer readers to Glover [27] and Habet [28].

In this section, a tabu search (NTS) algorithm that probabilistically accepts the second best solution in search process when a local optimum is reached is proposed to effectively solve the LIRP-FRL. The key components of the NTS, including a solution representation technique, an initial solution generation method, neighbourhood structures, and the general framework of the new tabu search, are presented in detail as follows.

3.1. Solution Representation. A good solution representation can not only describe the problem solutions clearly but also improve the performance of heuristics adopted. Similar to that of $\mathrm{Yu}$ et al. [29], in our implementation, solution $\left\{x_{1}, x_{2}, \ldots, x_{n}, x_{n+1}, \ldots, x_{n+m}\right\}$ is represented by a permutation of number $\{1,2, \ldots, n, n+1, n+2, \ldots, n+m\}$, where $x_{j} \in\{1,2, \ldots, n\}$ indexes customer $x_{j}$ and $x_{i} \in\{n+1, n+$ $2, \ldots, n+m\}$ denotes potential DC $x_{i}$.

An example was given in Figure 2. In this example, the first number is distribution center 21 , followed by distribution center 23. Since there are no customers between distribution center 21 and distribution center 23, distribution center 21 is closed. Customers $(19,16,15,14,8,11$, and 6) between distribution center 23 and distribution center 22 are real customers; thus distribution center 23 is opened to service these customers. The first route of distribution center 23 services customers $19,16,15$, and 14 . Because adding customer 8 exceeds the vehicle capacity, the second route services customers 8,11 , and 6 . Customer 6 is followed by distribution center 22, so the second route is terminated. For a similar reason as mentioned above, distribution centers 22 and 25 are open with two routes and one route, respectively.

This representation has determined which distribution center is open and the customers on each route. With that decided, we can easily calculate the objective function value, because the inventory cost is related to LRP, which means when LRP is solved the inventory cost can be uniquely determined by Proposition 2. Note that the capacity of distribution center is not taken into consideration. So during the decoding process, a per unit penalty cost $M$ with a big value is added to the objective function value when the total demand serviced by a distribution center exceeds its capacity.

To simplify the presentation of the algorithms proposed in following subsections, we say that an element $x_{i}$ of the solution is before another element $x_{j}$ or element $x_{j}$ is after element $x_{i}$ if and only if $i<j$. Further, with the smallest value $|i-j|$, if DC $x_{i}$ is before DC $x_{j}$, we say that DC $x_{i}$ is nearest before DC $x_{j}$, or DC $x_{j}$ is nearest after DC $x_{i}$.

3.2. Constructing Initial Solution. A greedy nearest neighbor method is proposed to construct an initial feasible solution of the LIRP-FRL, which is used as an input for the tabu search. Details of the greedy algorithm are given as follows.

Algorithm 3 (greedy nearest neighbour method).

Step 0 . Initialize $\Omega^{0}:=J, \Omega^{1}:=\varnothing, \Phi^{0}:=I$, and $\Phi^{1}:=\varnothing$.

Step 1. Assign each customer in $\Phi^{0}$ to its closest DCs in $\Omega^{0}$.

Step 2. Open the DC in $\Omega^{0}$ with the largest number of customers assigned to say $\mathrm{DC} j^{*}$.

Step 3. Sort the customers of set $\Phi^{0}$ in nondecreasing order of the distance between it and the $\mathrm{DC} j^{*}$.

Step 4. With the order, let $\Phi=\left\{s_{1}, s_{2}, \ldots, s_{\left|\Phi^{0}\right|}\right\}$ be the subset of customers of $\Phi^{0}$ with the largest $|\Phi|$ and $\sum_{i=1}^{|\Phi|} d_{s_{i}} \leq V_{j^{*}}$. Renew $\Omega^{1}:=\Omega^{1} \cup\left\{j^{*}\right\}, \Omega^{0}:=J / \Omega^{1}, \Phi^{1}:=\Phi^{1} \cup \Phi$, and $\Phi^{0}:=I / \Phi^{1}$.

Step 5. If $\Phi^{0} \neq \varnothing$, then return to Step 1; Otherwise, open the DCs in $\Omega^{1}$ and assign each customer to a DC according to the customer-DC assignments determined in Step 4.

Step 6. For each opened DC, sort the customers assigned to it into sequence by using a nearest neighbor heuristic (refer to Rosenkrantz et al. [30]). Split the sequence into several feasible routes so that vehicle capacity constraints are satisfied.

Step 7. Output feasible solution.

In our implementation of Step 2, if more than one DC has the largest number of customers assigned to, we select the DC with the highest capacity.

\subsection{Neighborhoods. Given a solution}

$$
X=\left(x_{1}, x_{2}, \ldots, x_{i-1}, x_{i}, x_{i+1}, \ldots, x_{j-1}, x_{j}, x_{j+1}, \ldots, x_{n}\right),
$$

a permutation of $\{1,2, \ldots, n, n+1, \ldots, n+m\}$, three kinds of neighbourhoods are considered in our tabu search. 
3.3.1. Insertion Neighbourhood. The insertion neighborhood is defined as the set of solutions that can be reached by insertion move, which deletes one element of the solution $X$ and then inserts it to another place. For example, delete element $x_{i}$ and insert it into the $j$ th place; we obtain a new solution:

$$
X^{\prime}=\left(x_{1}, x_{2}, \ldots, x_{i-1}, x_{i+1}, \ldots, x_{j-1}, x_{j}, x_{i}, x_{j+1}, \ldots, x_{n}\right) .
$$

According to the element selected (customer or depot) and the position it is inserted in, there are four cases:

(a) If $x_{i}$ and $x_{j}$ are both customers, then customer $x_{i}$ is reassigned to the DC that customer $x_{j}$ is assigned to.

(b) If $x_{i}$ and $x_{j}$ are both DCs, then reassign the customers previously assigned to the DC nearest before it. Close DC $x_{j}$ and assign its customers to DC $x_{i}$.

(c) If $x_{i}$ is a customer and $x_{j}$ is a DC, then customer $x_{i}$ is reassigned to $\mathrm{DC} x_{j}$.

(d) If $x_{i}$ is a DC and $x_{j}$ is a customer, reassign customers that are nearest after customer $x_{j}$ to DC $x_{i}$. All customers assigned to DC $x_{i}$ are reassigned to the DC nearest before it.

3.3.2. Swap Neighbourhood. The swap neighbourhood, a set of solutions, contains the solutions that can be reached by performing a swap move, which exchanges the places of two different elements of solution $X$. For example, exchange element $x_{i}$ and element $x_{j}$ of $X$; the resulting new solution is

$$
X^{\prime}=\left(x_{1}, x_{2}, \ldots, x_{i-1}, x_{j}, x_{i+1}, \ldots, x_{j-1}, x_{i}, x_{j+1}, \ldots, x_{n}\right) \text {. }
$$

Similar to the insert move, the swap move has also four cases:

(e) If $x_{i}$ and $x_{j}$ are both customers, then we exchange the two DCs they are currently assigned to and obtain two new customer-DC assignments.

(f) If $x_{i}$ and $x_{j}$ are both DCs, then we exchange all the customers currently assigned to them.

(g) If $x_{i}$ is a customer and $x_{j}$ is a DC, then the customers after $x_{i}$ assigned to the same DC are reassigned to DC $x_{j}$, and customer $x_{i}$ is reassigned to the DC nearest before DC $x_{j}$. All customers previously assigned to DC $x_{j}$ will be reassigned to the DC nearest before it.

(h) If $x_{i}$ is a DC and $x_{j}$ is a customer, this case is similar to case $g$.

3.3.3. 2-Opt Neighbourhood. With the consideration of vehicle capacity, in our definition, the 2-opt neighbourhood contains the solutions that can be obtained by selecting two customers of $X$ (e.g., $x_{i}$ and $x_{j}$ ) and then reversing the substring in the solution representation between them, the so-called 2opt move. For example, applying 2-opt move to elements $x_{i}$ and $x_{j}$, we obtain

$$
X^{\prime}=\left(x_{1}, x_{2}, \ldots, x_{i-1}, x_{j}, x_{j-1}, \ldots, x_{i+1}, x_{i}, x_{j+1}, \ldots, x_{n}\right) .
$$

3.4. Tabu Search Framework. To increase the probability of obtaining high quality solutions, a diversification strategy is implemented. This strategy probabilistically accepts the second best solution in the search process while no better solution can be found in the neighborhoods of the current solution. By doing so, we can effectively diversify the search direction and thus more solution space will be explored. Consequently, better solution may be found. The computation results in Section 4 prove this strategy. The proposed tabu search can be summarized as follows.

Algorithm 4 (new tabu search).

Step 0. Input an initial feasible solution $X_{0}$ (see Section 3.2) and initialize Iter $:=0, X_{\text {curr }}:=X_{0}$, and $\mathrm{Obj}^{*}:=\operatorname{Obj}\left(X_{0}\right)$.

Step 1. Explore the neighborhoods of $X_{\text {curr }}$ and denote the best solution in the neighborhoods, the best nontabu solution, and the second best nontabu solution as $X_{\text {best }}, X_{\text {tabu-best }}$, and $X_{\text {tabu-2nd }}$, respectively.

Step 2. If $\mathrm{Obj}\left(X_{\text {best }}\right)<\mathrm{Obj}^{*}$, then set $X_{\text {curr }}:=X_{\text {best }}$ and add the corresponding move to tabu list; otherwise,

if $\operatorname{Obj}\left(X_{\text {tabu-best }}\right)<\operatorname{Obj}\left(X_{\text {curr }}\right)$, then $X_{\text {curr }}:=X_{\text {tabu-best }}$ and add the corresponding move to tabu list;

otherwise, probabilistically move to $X_{\text {tabu-best }}$ or $X_{\text {tabu-2nd }}, X_{\text {curr }}:=\operatorname{Prob}\left\{X_{\text {tabu-best }}, X_{\text {tabu-2nd }}\right\}$, and add the corresponding move to tabu list.

Step 3. Iter := Iter +1 ; If no stopping criterion is reached, return to Step 1.

Step 4. Output the best solution objective function value $\mathrm{Obj}^{*}$.

In the proposed tabu search algorithm, Iter counts the number of iterations and $X_{0}$ represents the initial solution obtained by greedy nearest neighbour method (Section 3.2). $X_{\text {curr }}$ denotes the current solution. $\mathrm{Obj}^{*}$ is the objective function value corresponding to the best solution found so far. The function $\operatorname{Obj}(X)$ represents the objective function value of solution $X$.

In Step 2, comparing with the current solution $X_{\text {curr }}$ if no better solution can be found in its neighbourhood, we need to probabilistically move $X_{\text {curr }}$ to $X_{\text {tabu-best }}$ or $X_{\text {tabu-2nd }}$. In implementation, we randomly generate a value $\beta$ in interval $[0,1]$. If $\beta \geq \lambda$, then $X_{\text {curr }}$ is moved to $X_{\text {tabu-best }}$; and otherwise $X_{\text {curr }}$ is moved to $X_{\text {tabu-2nd }}$, where $\lambda$ is a given parameter.

At each iteration of the tabu search procedure, the best admissible nontabu insertion, swap or 2-opt move is performed. The two elements of solution $X_{\text {curr }}$ involved in such move are declared as tabu-active, which is forbidden to be removed in the next $t$ iterations. $t$ is the tabu-tenure parameter selected randomly from a range $\left[T_{\min }, T_{\max }\right]$.

The tabu search iteration is terminated if the maximum number of iterations maxIter is reached or if the best solution found so far has been improved in successive SucIter iteration. 
TABLE 1: Computational results on CLRP benchmarks.

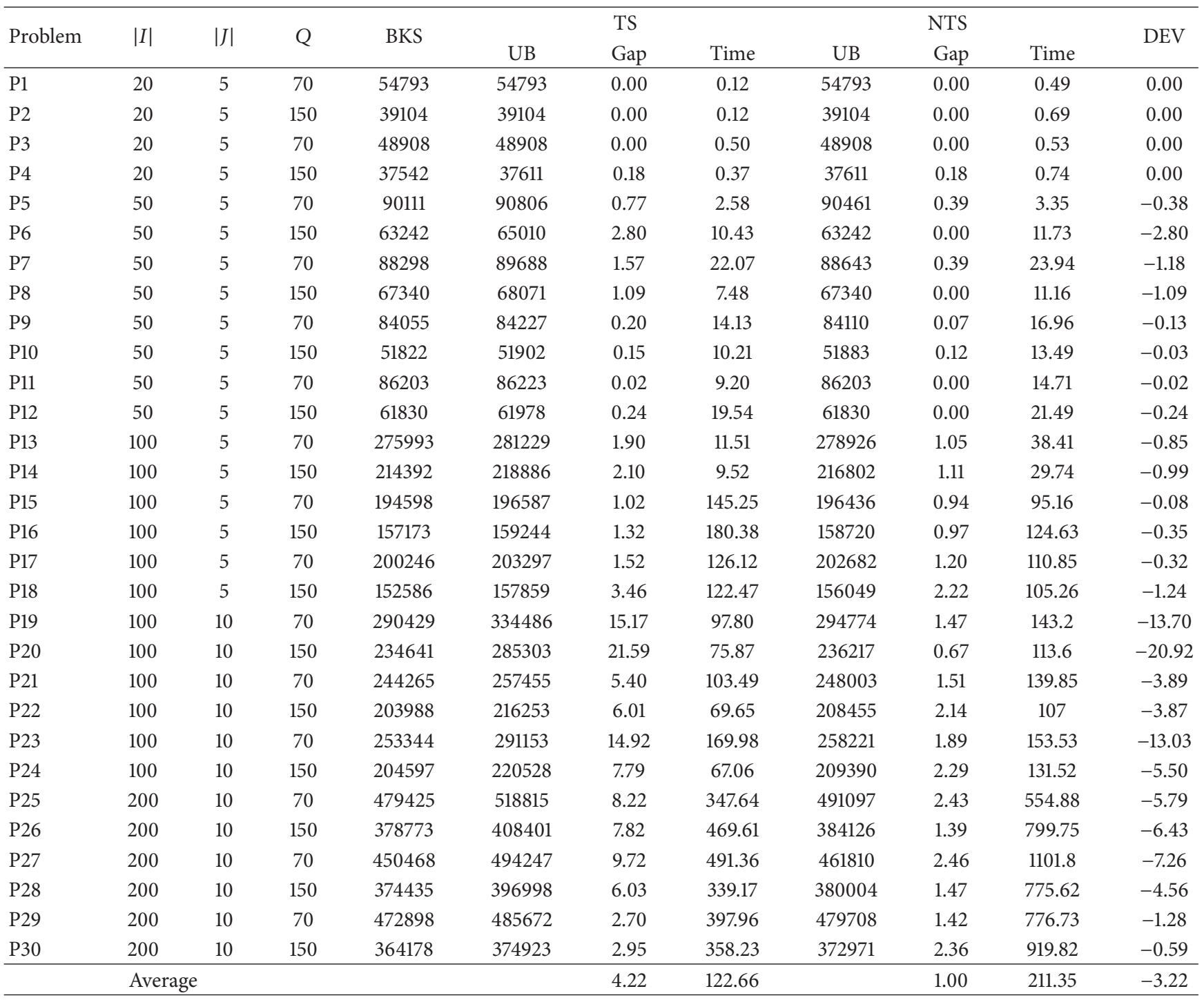

\section{Computational Results}

The proposed tabu search algorithm was coded in the $\mathrm{C}$ program and ran on a computer with Intel(R) Core(TM) CPU (3.2 GHz) with $4 \mathrm{~GB}$ of RAM under the Microsoft Windows 7 operation system.

4.1. Test Beds. Two test beds were used to evaluate the performance of the proposed tabu search algorithms:

(1) The benchmarks of CLRP (Prins et al. [31]), which contains 30 instances: two potential distribution center sizes $|J|=5$ or 10 are considered. The number of customers is $|I|=20,50,100$, and 200. The customers and DCs are uniformly distributed in a $[1,50] \times[1,50]$ square. The fixed opening costs of DCs are randomly generated from interval $[5000,125000]$. The traveling cost between two nodes equals their Euclidean distance multiplied by 100 and rounded up to the next integer. The setup cost of a vehicle is 1000 . The vehicle capacity $Q$ equals 70 or 150 . Customers' demands are randomly selected from interval $[11,20]$.

(2) Randomly generated LIRP-FRL instances: based on the data of CLRP benchmarks and using the setting of [25] for the parameters, such as the order cost of new products of DCs $A^{m}(=200)$, the order cost of recovered products of DCs $A^{r}(=100)$, the cost of manufacturing $c^{m}(=60)$, recovering $c^{r}(=50)$, disposal $c^{d}(-10)$, and the return rate of product, we randomly generate the LIRP-FRL instances as follows:

(i) The holding cost for each distribution center is uniformly distributed in $U[5,10]$.

(ii) The transportation cost per unit product of the distribution center at site $j, j \in J$, is uniformly distributed in $U[1,10]$. 
4.2. Parameter Settings. The maximum number of iterations for NTS maxiter is set to 10000 and the search iteration is terminated if the best upper bound has not been improved in successive 800 iteration (SusIter $=800$ ). Parameter $\lambda$ is uniformly distributed in $U[0.01,0.8]$. The lower and upper bound of tabu-tenure $T_{\min }$ and $T_{\max }$ are set to 5 and 12, respectively.

4.3. Numerical Results. To evaluate the performance of the proposed new tabu search (NTS), we compare it with the classical tabu search algorithm (denoted as TS in following discussion) in terms of the best upper bound (UB) found so far and the corresponding computational time in CPU seconds (time). Moreover, the parameter settings of TS is the same as NTS's except parameter $\lambda$ that TS did not have. Computational results on CLRP benchmarks are listed in Table 1. The column BKS is the best known solution objective function value reported in the literature. Gap is calculated by the following formula: Gap $=100 *(\mathrm{UB}-\mathrm{BKS}) / \mathrm{UB}$. DEV is the deviation of the gap of NTS from the gap of TS; that is, $\mathrm{DEV}=\mathrm{Gap}_{\mathrm{NTS}}-\mathrm{Gap}_{\mathrm{TS}}$.

Using the diversification strategy of probabilistically accepting the second best solution, the NTS increases the probability of exploring more solution space. As a result, the NTS takes more computational time than the TS. However, from Table 1, we can observe that although the average computational time of NTS is about two times that of TS, it is still in a very acceptable range. The NTS provides more competitive upper bound than the TS. The average gap between the best upper bound found so far by NTS and the best known upper bound in the literature is about $1.00 \%$, compared to the $4.22 \%$ of the TS. The NTS outperforms the TS in the quality of solutions obtained with the average deviation of the gap DEV $=-3.22 \%$.

Table 2 reports the computational results on test bed of randomly generated LIRP-FRL instances. From the table, we can also find that the NTS performs better than the classic TS with an average gap between the best upper bound of NTS and that of TS, about $-2.79 \%$. The average computational time of NTS is slightly bigger than that of TS.

4.4. Sensitivity Analysis. Sensitivity analysis is also conducted to investigate the effects on the total costs when the input parameters such as the return rate $(r)$ or the rate between the unit producing cost of new products and recovered products $\left(c^{m}: c^{r}\right)$ changes.

Table 3 reports the computational results when the product return rate is changed for each randomly generated instance. To manifest the trend more clearly, for each return rate value, we calculate the arithmetic average objective function values of all tested instances. The results are shown in Figure 3. From the figure, we can find that the total costs decrease with the increase of return rate $r$. Focusing on those products with high return rate can effectively reduce the system costs.

Table 4 shows the numerical results when the rate between the cost of producing a new product and that of recovering a damaged or old product varies. For each rate, we
TABLE 2: Computational results on LIRP-FRL instances.

\begin{tabular}{lcccccccc}
\hline \multirow{2}{*}{ Prob. ID $|I|$} & $|J|$ & Q & UB & Time & UB & Time & \\
& & & & TS Gap 1 $(\%)$ \\
\hline P1 & 20 & 5 & 70 & 67528 & 0.50 & 67528 & 0.53 & 0.00 \\
P2 & 20 & 5 & 150 & 42867 & 0.55 & 42867 & 0.61 & 0.00 \\
P3 & 20 & 5 & 70 & 60021 & 0.46 & 60021 & 0.65 & 0.00 \\
P4 & 20 & 5 & 150 & 40255 & 0.57 & 40255 & 0.62 & 0.00 \\
P5 & 50 & 5 & 70 & 115984 & 22.56 & 115534 & 22.95 & -0.39 \\
P6 & 50 & 5 & 150 & 84468 & 36.43 & 84401 & 34.18 & -0.08 \\
P7 & 50 & 5 & 70 & 117828 & 22.56 & 117530 & 24.06 & -0.25 \\
P8 & 50 & 5 & 150 & 96633 & 21.61 & 96161 & 21.64 & -0.49 \\
P9 & 50 & 5 & 70 & 112493 & 24.31 & 111625 & 24.51 & -0.78 \\
P10 & 50 & 5 & 150 & 82667 & 23.01 & 82392 & 24.66 & -0.33 \\
P11 & 50 & 5 & 70 & 108639 & 21.28 & 108568 & 20.32 & -0.07 \\
P12 & 50 & 5 & 150 & 82938 & 21.58 & 82804 & 22.07 & -0.16 \\
P13 & 100 & 5 & 70 & 342786 & 139.24 & 342309 & 139.97 & -0.14 \\
P14 & 100 & 5 & 150 & 276104 & 137.64 & 273409 & 138.72 & -1.37 \\
P15 & 100 & 5 & 70 & 239544 & 143.75 & 238937 & 158.07 & -0.25 \\
P16 & 100 & 5 & 150 & 201533 & 125.58 & 200708 & 135.06 & -0.41 \\
P17 & 100 & 5 & 70 & 250239 & 121.28 & 249565 & 124.88 & -0.27 \\
P18 & 100 & 5 & 150 & 198842 & 126.84 & 197310 & 128.30 & -0.78 \\
P19 & 100 & 10 & 70 & 406342 & 138.30 & 396469 & 161.50 & -2.49 \\
P20 & 100 & 10 & 150 & 368668 & 138.84 & 339644 & 157.86 & -8.55 \\
P21 & 100 & 10 & 70 & 328174 & 121.59 & 320363 & 151.15 & -2.44 \\
P22 & 100 & 10 & 150 & 278729 & 128.50 & 274026 & 134.76 & -1.72 \\
P23 & 100 & 10 & 70 & 388259 & 113.47 & 320651 & 143.94 & -21.08 \\
P24 & 100 & 10 & 150 & 339851 & 120.77 & 283008 & 136.13 & -20.09 \\
P25 & 200 & 10 & 70 & 645014 & 393.75 & 629604 & 525.43 & -2.45 \\
P26 & 200 & 10 & 150 & 558189 & 368.91 & 528721 & 510.52 & -5.57 \\
P27 & 200 & 10 & 70 & 627737 & 427.91 & 611151 & 542.92 & -2.71 \\
P28 & 200 & 10 & 150 & 543988 & 379.41 & 517132 & 432.50 & -5.19 \\
P29 & 200 & 10 & 70 & 607894 & 419.25 & 598889 & 428.33 & -1.50 \\
P30 & 200 & 10 & 150 & 530675 & 422.72 & 509796 & 563.14 & -4.10 \\
\hline & Average & & & 138.77 & & 163.67 & -2.79 \\
\hline
\end{tabular}

${ }^{*}$ Gap $1(\%)=100 *(\mathrm{H} 2$ solution value $-\mathrm{H} 1$ solution value $) / \mathrm{H} 2$ solution value.

average the best upper bound obtained for all tested instances. The results are depicted in Figure 4. From the figure, we can observe that the total costs decrease with the increase of the rate. That means reducing the unit recovering cost of product can effectively reduce the total costs, which indicates us to retrieve those subnew products or to improve repairing technique so as to reduce recovering cost.

\section{Conclusions and Future Works}

We study a location-inventory-routing problem in forward and reverse logistics (LIRP-FRL) in this paper. A nonlinear mixed integer programming model is proposed to formulate the LIRP-FRL. Based on this model, a new tabu search that probabilistically accepts the second best solution in its neighborhoods is proposed to find near optimal solution for the problem. Numerical experiments on CLRP benchmarks 
TABLE 3: Results of different return rates.

\begin{tabular}{|c|c|c|c|c|c|c|c|c|c|}
\hline \multirow{2}{*}{ Prob. ID } & \multirow{2}{*}{$r$} & \multirow{2}{*}{$|I|$} & \multirow{2}{*}{$|J|$} & \multirow{2}{*}{$Q$} & \multicolumn{2}{|c|}{ TS } & \multicolumn{2}{|c|}{ NTS } & \multirow{2}{*}{ Gap 1 (\%) } \\
\hline & & & & & UB & Time & UB & Time & \\
\hline \multirow{9}{*}{ P1 } & 0.1 & \multirow{9}{*}{20} & \multirow{9}{*}{5} & \multirow{9}{*}{70} & 67617 & 3.22 & 67617 & 2.66 & 0.00 \\
\hline & 0.2 & & & & 67522 & 3.38 & 67522 & 3.48 & 0.00 \\
\hline & 0.3 & & & & 67423 & 3.64 & 67423 & 3.14 & 0.00 \\
\hline & 0.4 & & & & 67568 & 2.73 & 67320 & 3.28 & -0.37 \\
\hline & 0.5 & & & & 67293 & 4.69 & 67211 & 3.22 & -0.12 \\
\hline & 0.6 & & & & 67094 & 3.26 & 67094 & 2.95 & 0.00 \\
\hline & 0.7 & & & & 66966 & 3.19 & 66966 & 2.77 & 0.00 \\
\hline & 0.8 & & & & 66904 & 3.15 & 66822 & 3.67 & -0.12 \\
\hline & 0.9 & & & & 66646 & 2.68 & 66646 & 3.15 & 0.00 \\
\hline \multirow{9}{*}{ P2 } & 0.1 & \multirow{9}{*}{20} & \multirow{9}{*}{5} & \multirow{9}{*}{150} & 42972 & 2.54 & 42972 & 2.87 & 0.00 \\
\hline & 0.2 & & & & 42969 & 2.43 & 42969 & 2.96 & 0.00 \\
\hline & 0.3 & & & & 42965 & 3.07 & 42965 & 2.57 & 0.00 \\
\hline & 0.4 & & & & 42961 & 3.40 & 42961 & 2.66 & 0.00 \\
\hline & 0.5 & & & & 42955 & 3.00 & 42955 & 3.18 & 0.00 \\
\hline & 0.6 & & & & 42948 & 2.63 & 42948 & 2.92 & 0.00 \\
\hline & 0.7 & & & & 42939 & 2.68 & 42939 & 3.08 & 0.00 \\
\hline & 0.8 & & & & 42927 & 2.66 & 42927 & 3.02 & 0.00 \\
\hline & 0.9 & & & & 42908 & 2.47 & 42908 & 3.04 & 0.00 \\
\hline & 0.1 & & & & 61544 & 2.70 & 61296 & 2.68 & -0.40 \\
\hline & 0.2 & & & & 61175 & 3.14 & 61175 & 3.40 & 0.00 \\
\hline & 0.3 & & & & 61050 & 2.68 & 61050 & 2.99 & 0.00 \\
\hline & 0.4 & & & & 61011 & 3.16 & 60921 & 2.91 & -0.15 \\
\hline P3 & 0.5 & 20 & 5 & 70 & 60786 & 2.62 & 60786 & 2.98 & 0.00 \\
\hline & 0.6 & & & & 60733 & 2.55 & 60644 & 2.81 & -0.15 \\
\hline & 0.7 & & & & 60492 & 2.99 & 60492 & 3.23 & 0.00 \\
\hline & 0.8 & & & & 60325 & 2.87 & 60325 & 3.10 & 0.00 \\
\hline & 0.9 & & & & 60308 & 3.18 & 60127 & 3.19 & -0.30 \\
\hline & 0.1 & & & & 40396 & 2.56 & 40396 & 2.71 & 0.00 \\
\hline & 0.2 & & & & 40395 & 2.58 & 40395 & 2.63 & 0.00 \\
\hline & 0.3 & & & & 40393 & 2.64 & 40393 & 2.94 & 0.00 \\
\hline & 0.4 & & & & 40390 & 2.68 & 40390 & 2.41 & 0.00 \\
\hline $\mathrm{P} 4$ & 0.5 & 20 & 5 & 150 & 40386 & 2.66 & 40386 & 3.12 & 0.00 \\
\hline & 0.6 & & & & 40381 & 2.53 & 40381 & 2.46 & 0.00 \\
\hline & 0.7 & & & & 40373 & 2.68 & 40373 & 2.66 & 0.00 \\
\hline & 0.8 & & & & 40363 & 2.46 & 40363 & 2.63 & 0.00 \\
\hline & 0.9 & & & & 40346 & 2.88 & 40346 & 2.61 & 0.00 \\
\hline & 0.1 & & & & 117680 & 36.19 & 116409 & 34.62 & -1.08 \\
\hline & 0.2 & & & & 115813 & 32.65 & 115115 & 31.58 & -0.60 \\
\hline & 0.3 & & & & 114965 & 30.64 & 114542 & 31.43 & -0.37 \\
\hline & 0.4 & & & & 115279 & 30.7 & 114927 & 31.34 & -0.31 \\
\hline P5 & 0.5 & 50 & 5 & 70 & 116446 & 26.22 & 115078 & 30.92 & -1.17 \\
\hline & 0.6 & & & & 114151 & 30.48 & 114039 & 31.18 & -0.10 \\
\hline & 0.7 & & & & 114504 & 31.34 & 114190 & 32.04 & -0.27 \\
\hline & 0.8 & & & & 115578 & 28.66 & 114239 & 26.56 & -1.16 \\
\hline & 0.9 & & & & 112326 & 31.31 & 112310 & 33.62 & -0.01 \\
\hline & 0.1 & & & & 87885 & 20.30 & 87678 & 24.04 & -0.24 \\
\hline & 0.2 & & & & 87289 & 23.82 & 86748 & 27.87 & -0.62 \\
\hline & 0.3 & & & & 88191 & 23.95 & 87140 & 24.59 & -1.19 \\
\hline & 0.4 & & & & 89497 & 20.47 & 86970 & 23.87 & -2.82 \\
\hline P6 & 0.5 & 50 & 5 & 150 & 87240 & 21.81 & 86955 & 24.84 & -0.33 \\
\hline & 0.6 & & & & 87233 & 24.34 & 86283 & 24.26 & -1.09 \\
\hline & 0.7 & & & & 86366 & 24.17 & 86173 & 21.05 & -0.22 \\
\hline & 0.8 & & & & 85494 & 22.81 & 84771 & 24.78 & -0.85 \\
\hline & 0.9 & & & & 84673 & 23.93 & 84358 & 24.24 & -0.37 \\
\hline
\end{tabular}


TABle 3: Continued.

\begin{tabular}{|c|c|c|c|c|c|c|c|c|c|}
\hline \multirow{2}{*}{ Prob. ID } & \multirow{2}{*}{$r$} & \multirow{2}{*}{$|I|$} & \multirow{2}{*}{$|J|$} & \multirow{2}{*}{$Q$} & \multicolumn{2}{|c|}{ TS } & \multicolumn{2}{|c|}{ NTS } & \multirow{2}{*}{ Gap $1(\%)$} \\
\hline & & & & & UB & Time & UB & Time & \\
\hline \multirow{9}{*}{ P7 } & 0.1 & \multirow{9}{*}{50} & \multirow{9}{*}{5} & \multirow{9}{*}{70} & 122232 & 26.84 & 121605 & 22.23 & -0.51 \\
\hline & 0.2 & & & & 121038 & 26.14 & 120826 & 26.16 & -0.18 \\
\hline & 0.3 & & & & 121147 & 26.17 & 120530 & 26.66 & -0.51 \\
\hline & 0.4 & & & & 120239 & 26.41 & 119945 & 26.86 & -0.24 \\
\hline & 0.5 & & & & 120223 & 26.11 & 119788 & 26.03 & -0.36 \\
\hline & 0.6 & & & & 119636 & 26.05 & 119545 & 28.81 & -0.08 \\
\hline & 0.7 & & & & 119795 & 25.50 & 119440 & 32.04 & -0.30 \\
\hline & 0.8 & & & & 119994 & 26.05 & 119100 & 27.80 & -0.75 \\
\hline & 0.9 & & & & 119728 & 25.72 & 118222 & 27.31 & -1.26 \\
\hline \multirow{9}{*}{ P8 } & 0.1 & \multirow{9}{*}{50} & \multirow{9}{*}{5} & \multirow{9}{*}{150} & 99470 & 26.38 & 99178 & 27.75 & -0.29 \\
\hline & 0.2 & & & & 99455 & 25.21 & 99319 & 25.61 & -0.14 \\
\hline & 0.3 & & & & 100163 & 28.65 & 98682 & 21.78 & -1.48 \\
\hline & 0.4 & & & & 98722 & 25.14 & 98642 & 25.32 & -0.08 \\
\hline & 0.5 & & & & 98930 & 25.27 & 98617 & 27.10 & -0.32 \\
\hline & 0.6 & & & & 98131 & 24.90 & 98056 & 26.23 & -0.08 \\
\hline & 0.7 & & & & 98723 & 25.60 & 97815 & 23.55 & -0.92 \\
\hline & 0.8 & & & & 97633 & 25.78 & 97296 & 33.13 & -0.35 \\
\hline & 0.9 & & & & 97752 & 25.43 & 96882 & 26.07 & -0.89 \\
\hline & 0.1 & & & & 86438 & 25.22 & 86398 & 29.74 & -0.05 \\
\hline & 0.2 & & & & 86031 & 22.20 & 85926 & 28.47 & -0.12 \\
\hline & 0.3 & & & & 86300 & 25.01 & 85635 & 21.50 & -0.77 \\
\hline & 0.4 & & & & 85292 & 21.61 & 85283 & 22.87 & -0.01 \\
\hline P9 & 0.5 & 50 & 5 & 70 & 85084 & 24.46 & 84834 & 28.05 & -0.29 \\
\hline & 0.6 & & & & 84628 & 23.10 & 84495 & 28.72 & -0.16 \\
\hline & 0.7 & & & & 84358 & 24.98 & 84116 & 22.87 & -0.29 \\
\hline & 0.8 & & & & 83239 & 21.88 & 83103 & 21.64 & -0.16 \\
\hline & 0.9 & & & & 83386 & 22.48 & 82806 & 25.97 & -0.70 \\
\hline & 0.1 & & & & 115321 & 25.76 & 115143 & 28.26 & -0.15 \\
\hline & 0.2 & & & & 114968 & 26.16 & 114609 & 21.55 & -0.31 \\
\hline & 0.3 & & & & 114628 & 27.12 & 114347 & 24.59 & -0.25 \\
\hline & 0.4 & & & & 114620 & 26.62 & 113993 & 27.86 & -0.55 \\
\hline P10 & 0.5 & 50 & 5 & 150 & 114243 & 25.93 & 113821 & 27.95 & -0.37 \\
\hline & 0.6 & & & & 113720 & 26.05 & 113461 & 29.29 & -0.23 \\
\hline & 0.7 & & & & 113367 & 22.95 & 113204 & 28.36 & -0.14 \\
\hline & 0.8 & & & & 113157 & 26.73 & 112657 & 28.41 & -0.44 \\
\hline & 0.9 & & & & 112518 & 32.52 & 111908 & 26.90 & -0.54 \\
\hline & 0.1 & & & & 112376 & 26.82 & 111843 & 32.35 & -0.47 \\
\hline & 0.2 & & & & 111582 & 25.94 & 111286 & 27.18 & -0.27 \\
\hline & 0.3 & & & & 111920 & 23.13 & 111054 & 29.51 & -0.77 \\
\hline & 0.4 & & & & 111642 & 24.91 & 110986 & 28.05 & -0.59 \\
\hline P11 & 0.5 & 50 & 5 & 70 & 111345 & 23.08 & 110891 & 27.75 & -0.41 \\
\hline & 0.6 & & & & 110712 & 26.11 & 110203 & 28.93 & -0.46 \\
\hline & 0.7 & & & & 110789 & 25.79 & 109949 & 29.03 & -0.76 \\
\hline & 0.8 & & & & 110232 & 25.79 & 109923 & 28.18 & -0.28 \\
\hline & 0.9 & & & & 109797 & 24.80 & 109042 & 32.89 & -0.69 \\
\hline & 0.1 & & & & 85505 & 24.69 & 85246 & 27.45 & -0.30 \\
\hline & 0.2 & & & & 85187 & 24.25 & 85019 & 23.92 & -0.20 \\
\hline & 0.3 & & & & 84879 & 24.73 & 84757 & 24.78 & -0.14 \\
\hline & 0.4 & & & & 84797 & 28.33 & 84337 & 30.83 & -0.54 \\
\hline P12 & 0.5 & 50 & 5 & 150 & 84312 & 25.84 & 83955 & 28.38 & -0.42 \\
\hline & 0.6 & & & & 84053 & 26.65 & 83933 & 21.82 & -0.14 \\
\hline & 0.7 & & & & 83933 & 26.31 & 83358 & 31.33 & -0.69 \\
\hline & 0.8 & & & & 83651 & 26.92 & 82897 & 29.92 & -0.90 \\
\hline & 0.9 & & & & 82655 & 23.22 & 82439 & 26.95 & -0.26 \\
\hline
\end{tabular}


TABle 3: Continued.

\begin{tabular}{|c|c|c|c|c|c|c|c|c|c|}
\hline \multirow{2}{*}{ Prob. ID } & \multirow{2}{*}{$r$} & \multirow{2}{*}{$|I|$} & \multirow{2}{*}{$|J|$} & \multirow{2}{*}{ Q } & \multicolumn{2}{|c|}{ TS } & \multicolumn{2}{|c|}{ NTS } & \multirow{2}{*}{ Gap $1(\%)$} \\
\hline & & & & & UB & Time & UB & Time & \\
\hline \multirow{9}{*}{$\mathrm{P} 13$} & 0.1 & \multirow{9}{*}{100} & \multirow{9}{*}{5} & \multirow{9}{*}{70} & 343359 & 189.12 & 341812 & 146.89 & -0.45 \\
\hline & 0.2 & & & & 346569 & 187.44 & 343870 & 187.75 & -0.78 \\
\hline & 0.3 & & & & 345210 & 169.49 & 342124 & 141.65 & -0.89 \\
\hline & 0.4 & & & & 348100 & 180.08 & 345668 & 190.9 & -0.70 \\
\hline & 0.5 & & & & 349269 & 159.25 & 343318 & 184.88 & -1.70 \\
\hline & 0.6 & & & & 347719 & 182.23 & 343891 & 162.19 & -1.10 \\
\hline & 0.7 & & & & 349525 & 182.84 & 342643 & 169.88 & -1.97 \\
\hline & 0.8 & & & & 349171 & 160.17 & 341151 & 158.68 & -2.30 \\
\hline & 0.9 & & & & 351251 & 182.08 & 349382 & 186.92 & -0.53 \\
\hline \multirow{9}{*}{ P14 } & 0.1 & \multirow{9}{*}{100} & \multirow{9}{*}{5} & \multirow{9}{*}{150} & 282054 & 135.84 & 281389 & 190.02 & -0.24 \\
\hline & 0.2 & & & & 281199 & 128.94 & 279541 & 151.39 & -0.59 \\
\hline & 0.3 & & & & 279903 & 155.98 & 279153 & 160.32 & -0.27 \\
\hline & 0.4 & & & & 278748 & 161.52 & 277988 & 130.87 & -0.27 \\
\hline & 0.5 & & & & 279806 & 152.32 & 278460 & 162.29 & -0.48 \\
\hline & 0.6 & & & & 280003 & 188.32 & 279155 & 127.99 & -0.30 \\
\hline & 0.7 & & & & 280823 & 126.66 & 278542 & 171.61 & -0.81 \\
\hline & 0.8 & & & & 276206 & 136.94 & 275974 & 138.12 & -0.08 \\
\hline & 0.9 & & & & 276528 & 157.65 & 275917 & 163.14 & -0.22 \\
\hline & 0.1 & & & & 246010 & 156.28 & 245337 & 148.54 & -0.27 \\
\hline & 0.2 & & & & 245120 & 175.84 & 244537 & 172.42 & -0.24 \\
\hline & 0.3 & & & & 244563 & 154.52 & 243754 & 154.32 & -0.33 \\
\hline & 0.4 & & & & 243537 & 154.53 & 243124 & 133.88 & -0.17 \\
\hline P15 & 0.5 & 100 & 5 & 70 & 242570 & 120.21 & 241529 & 192.88 & -0.43 \\
\hline & 0.6 & & & & 241778 & 171.75 & 241435 & 150.70 & -0.14 \\
\hline & 0.7 & & & & 240726 & 153.04 & 240237 & 138.58 & -0.20 \\
\hline & 0.8 & & & & 239819 & 149.01 & 239716 & 153.79 & -0.04 \\
\hline & 0.9 & & & & 238793 & 145.75 & 238624 & 144.34 & -0.07 \\
\hline & 0.1 & & & & 209027 & 131.28 & 208711 & 153.17 & -0.15 \\
\hline & 0.2 & & & & 208060 & 149.78 & 207229 & 157.39 & -0.40 \\
\hline & 0.3 & & & & 206328 & 167.99 & 205454 & 200.44 & -0.42 \\
\hline & 0.4 & & & & 207220 & 154.39 & 205877 & 195.70 & -0.65 \\
\hline P16 & 0.5 & 100 & 5 & 150 & 206676 & 163.29 & 205654 & 172.25 & -0.49 \\
\hline & 0.6 & & & & 204833 & 137.78 & 204547 & 168.87 & -0.14 \\
\hline & 0.7 & & & & 204402 & 137.86 & 204284 & 153.95 & -0.06 \\
\hline & 0.8 & & & & 203509 & 173.04 & 203068 & 191.72 & -0.22 \\
\hline & 0.9 & & & & 203695 & 153.95 & 201648 & 146.62 & -1.00 \\
\hline & 0.1 & & & & 259816 & 195.04 & 258025 & 149.48 & -0.69 \\
\hline & 0.2 & & & & 258004 & 164.99 & 256937 & 193.31 & -0.41 \\
\hline & 0.3 & & & & 256120 & 124.51 & 256057 & 123.60 & -0.02 \\
\hline & 0.4 & & & & 254658 & 130.21 & 254594 & 150.79 & -0.03 \\
\hline P17 & 0.5 & 100 & 5 & 70 & 254764 & 133.42 & 253722 & 165.32 & -0.41 \\
\hline & 0.6 & & & & 255047 & 155.20 & 252587 & 151.01 & -0.96 \\
\hline & 0.7 & & & & 250732 & 153.72 & 249812 & 149.22 & -0.37 \\
\hline & 0.8 & & & & 253732 & 182.23 & 249612 & 167.72 & -1.62 \\
\hline & 0.9 & & & & 252906 & 168.49 & 249337 & 157.79 & -1.41 \\
\hline & 0.1 & & & & 204826 & 145.28 & 204678 & 191.50 & -0.07 \\
\hline & 0.2 & & & & 205474 & 172.75 & 204621 & 193.28 & -0.42 \\
\hline & 0.3 & & & & 203254 & 167.53 & 202691 & 155.11 & -0.28 \\
\hline & 0.4 & & & & 203120 & 148.82 & 201380 & 121.34 & -0.86 \\
\hline P18 & 0.5 & 100 & 5 & 150 & 202003 & 122.00 & 201329 & 145.70 & -0.33 \\
\hline & 0.6 & & & & 200401 & 145.62 & 199858 & 151.80 & -0.27 \\
\hline & 0.7 & & & & 199032 & 147.58 & 198618 & 155.51 & -0.21 \\
\hline & 0.8 & & & & 198858 & 153.76 & 197776 & 145.95 & -0.54 \\
\hline & 0.9 & & & & 199502 & 134.57 & 197513 & 150.85 & -1.00 \\
\hline
\end{tabular}


TABle 3: Continued.

\begin{tabular}{|c|c|c|c|c|c|c|c|c|c|}
\hline \multirow{2}{*}{ Prob. ID } & \multirow{2}{*}{$r$} & \multirow{2}{*}{$|I|$} & \multirow{2}{*}{$|J|$} & \multirow{2}{*}{$Q$} & \multicolumn{2}{|c|}{ TS } & \multicolumn{2}{|c|}{ NTS } & \multirow{2}{*}{ Gap $1(\%)$} \\
\hline & & & & & UB & Time & UB & Time & \\
\hline \multirow{9}{*}{ P19 } & 0.1 & \multirow{9}{*}{100} & \multirow{9}{*}{10} & \multirow{9}{*}{70} & 414613 & 171.01 & 401365 & 198.5 & -3.20 \\
\hline & 0.2 & & & & 413680 & 164.18 & 400546 & 204.34 & -3.17 \\
\hline & 0.3 & & & & 420538 & 167.47 & 399896 & 195.42 & -4.91 \\
\hline & 0.4 & & & & 414460 & 154.11 & 394054 & 202.68 & -4.92 \\
\hline & 0.5 & & & & 411688 & 158.96 & 395828 & 211.36 & -3.85 \\
\hline & 0.6 & & & & 412339 & 162.17 & 394750 & 213.73 & -4.27 \\
\hline & 0.7 & & & & 410641 & 163.97 & 394617 & 210.72 & -3.90 \\
\hline & 0.8 & & & & 409561 & 181.48 & 394513 & 218.27 & -3.67 \\
\hline & 0.9 & & & & 408967 & 178.1 & 394294 & 201.13 & -3.59 \\
\hline \multirow{9}{*}{ P20 } & 0.1 & \multirow{9}{*}{100} & \multirow{9}{*}{10} & \multirow{9}{*}{150} & 365982 & 113.65 & 362144 & 114.33 & -1.05 \\
\hline & 0.2 & & & & 365164 & 111.79 & 361157 & 142.29 & -1.10 \\
\hline & 0.3 & & & & 365868 & 117.97 & 349578 & 113.22 & -4.45 \\
\hline & 0.4 & & & & 365307 & 145.30 & 349277 & 144.62 & -4.39 \\
\hline & 0.5 & & & & 366266 & 115.01 & 348378 & 158.55 & -4.88 \\
\hline & 0.6 & & & & 363989 & 151.05 & 348198 & 117.37 & -4.34 \\
\hline & 0.7 & & & & 113367 & 22.95 & 113204 & 28.36 & -0.14 \\
\hline & 0.8 & & & & 113157 & 26.73 & 112657 & 28.41 & -0.44 \\
\hline & 0.9 & & & & 112518 & 32.52 & 111908 & 26.90 & -0.54 \\
\hline & 0.1 & & & & 330001 & 132.05 & 323564 & 131.91 & -1.95 \\
\hline & 0.2 & & & & 329657 & 155.75 & 322848 & 178.39 & -2.07 \\
\hline & 0.3 & & & & 329303 & 166.30 & 322162 & 176.31 & -2.17 \\
\hline & 0.4 & & & & 328939 & 160.91 & 321756 & 167.07 & -2.18 \\
\hline P21 & 0.5 & 100 & 10 & 70 & 328561 & 167.71 & 321589 & 152.01 & -2.12 \\
\hline & 0.6 & & & & 328166 & 166.06 & 321183 & 182.85 & -2.13 \\
\hline & 0.7 & & & & 327744 & 132.12 & 321102 & 174.83 & -2.03 \\
\hline & 0.8 & & & & 327281 & 133.30 & 320160 & 179.93 & -2.18 \\
\hline & 0.9 & & & & 326742 & 134.59 & 319244 & 188.47 & -2.29 \\
\hline & 0.1 & & & & 281700 & 140.71 & 278464 & 129.13 & -1.15 \\
\hline & 0.2 & & & & 285498 & 140.01 & 278181 & 179.95 & -2.56 \\
\hline & 0.3 & & & & 285035 & 128.87 & 277760 & 184.58 & -2.55 \\
\hline & 0.4 & & & & 282821 & 157.09 & 277349 & 159.42 & -1.93 \\
\hline P22 & 0.5 & 100 & 10 & 150 & 284563 & 129.31 & 277133 & 181.07 & -2.61 \\
\hline & 0.6 & & & & 284057 & 152.07 & 276776 & 183.34 & -2.56 \\
\hline & 0.7 & & & & 280960 & 129.57 & 276530 & 172.01 & -1.58 \\
\hline & 0.8 & & & & 282955 & 157.32 & 276208 & 181.27 & -2.38 \\
\hline & 0.9 & & & & 278306 & 132.02 & 273261 & 178.52 & -1.81 \\
\hline & 0.1 & & & & 399113 & 125.10 & 375576 & 164.77 & -5.90 \\
\hline & 0.2 & & & & 396761 & 146.84 & 371533 & 160.97 & -6.36 \\
\hline & 0.3 & & & & 397280 & 168.97 & 360348 & 155.58 & -9.30 \\
\hline & 0.4 & & & & 396341 & 139.25 & 347788 & 175.99 & -12.25 \\
\hline P23 & 0.5 & 100 & 10 & 70 & 393942 & 143.91 & 346723 & 183.96 & -11.99 \\
\hline & 0.6 & & & & 392957 & 153.30 & 344273 & 145.58 & -12.39 \\
\hline & 0.7 & & & & 393378 & 159.10 & 325508 & 172.95 & -17.25 \\
\hline & 0.8 & & & & 390858 & 130.43 & 325392 & 172.15 & -16.75 \\
\hline & 0.9 & & & & 391111 & 140.30 & 322910 & 167.81 & -17.44 \\
\hline & 0.1 & & & & 297873 & 126.28 & 296530 & 138.04 & -0.45 \\
\hline & 0.2 & & & & 295735 & 139.68 & 294394 & 164.50 & -0.45 \\
\hline & 0.3 & & & & 334584 & 138.60 & 292118 & 173.75 & -12.69 \\
\hline & 0.4 & & & & 334011 & 152.64 & 288251 & 183.57 & -13.70 \\
\hline P24 & 0.5 & 100 & 10 & 150 & 345871 & 130.23 & 287245 & 142.65 & -16.95 \\
\hline & 0.6 & & & & 343622 & 150.07 & 286258 & 149.21 & -16.69 \\
\hline & 0.7 & & & & 344063 & 132.18 & 285833 & 132.30 & -16.92 \\
\hline & 0.8 & & & & 343388 & 151.00 & 285505 & 145.31 & -16.86 \\
\hline & 0.9 & & & & 342624 & 161.02 & 276622 & 165.18 & -19.26 \\
\hline
\end{tabular}


TABle 3: Continued.

\begin{tabular}{|c|c|c|c|c|c|c|c|c|c|}
\hline \multirow{2}{*}{ Prob. ID } & \multirow{2}{*}{$r$} & \multirow{2}{*}{$|I|$} & \multirow{2}{*}{$|J|$} & \multirow{2}{*}{$Q$} & \multicolumn{2}{|c|}{ TS } & \multicolumn{2}{|c|}{ NTS } & \multirow{2}{*}{ Gap 1 (\%) } \\
\hline & & & & & UB & Time & UB & Time & \\
\hline \multirow{9}{*}{ P25 } & 0.1 & \multirow{9}{*}{200} & \multirow{9}{*}{10} & \multirow{9}{*}{70} & 665405 & 1003.37 & 646837 & 1358.56 & -2.79 \\
\hline & 0.2 & & & & 663368 & 1022.24 & 646011 & 1275.81 & -2.62 \\
\hline & 0.3 & & & & 662357 & 840.78 & 645933 & 1052.16 & -2.48 \\
\hline & 0.4 & & & & 660795 & 819.73 & 643790 & 900.59 & -2.57 \\
\hline & 0.5 & & & & 660155 & 819.55 & 641200 & 1170.31 & -2.87 \\
\hline & 0.6 & & & & 657577 & 835.74 & 640619 & 1172.54 & -2.58 \\
\hline & 0.7 & & & & 655423 & 831.23 & 640582 & 851.98 & -2.26 \\
\hline & 0.8 & & & & 654409 & 830.95 & 637338 & 858.12 & -2.61 \\
\hline & 0.9 & & & & 653286 & 792.33 & 622908 & 836.45 & -4.65 \\
\hline \multirow{9}{*}{ P26 } & 0.1 & \multirow{9}{*}{200} & \multirow{9}{*}{10} & \multirow{9}{*}{150} & 573667 & 753.47 & 553421 & 1069.24 & -3.53 \\
\hline & 0.2 & & & & 570023 & 765.06 & 551891 & 1297.82 & -3.18 \\
\hline & 0.3 & & & & 569666 & 776.15 & 537807 & 1069.80 & -5.59 \\
\hline & 0.4 & & & & 567939 & 797.95 & 535782 & 1163.39 & -5.66 \\
\hline & 0.5 & & & & 564777 & 900.56 & 535225 & 1108.99 & -5.23 \\
\hline & 0.6 & & & & 564413 & 887.18 & 535083 & 1195.13 & -5.20 \\
\hline & 0.7 & & & & 562167 & 849.13 & 531505 & 1236.66 & -5.45 \\
\hline & 0.8 & & & & 560098 & 799.95 & 514584 & 1157.03 & -8.13 \\
\hline & 0.9 & & & & 557554 & 864.90 & 513652 & 1023.90 & -7.87 \\
\hline & 0.1 & & & & 661785 & 1384.45 & 631700 & 1228.42 & -4.55 \\
\hline & 0.2 & & & & 662602 & 1317.90 & 630853 & 969.39 & -4.79 \\
\hline & 0.3 & & & & 647333 & 1144.41 & 630036 & 1119.12 & -2.67 \\
\hline & 0.4 & & & & 647377 & 1238.22 & 625782 & 1060.19 & -3.34 \\
\hline P27 & 0.5 & 200 & 10 & 70 & 647837 & 955.81 & 625659 & 1159.76 & -3.42 \\
\hline & 0.6 & & & & 651234 & 972.11 & 624175 & 1104.26 & -4.16 \\
\hline & 0.7 & & & & 643657 & 994.18 & 623635 & 1203.08 & -3.11 \\
\hline & 0.8 & & & & 648507 & 1007.51 & 618926 & 1251.66 & -4.56 \\
\hline & 0.9 & & & & 640631 & 974.55 & 617253 & 1352.17 & -3.65 \\
\hline & 0.1 & & & & 560151 & 1039.68 & 554838 & 901.12 & -0.95 \\
\hline & 0.2 & & & & 567066 & 805.96 & 552250 & 1205.61 & -2.61 \\
\hline & 0.3 & & & & 565177 & 1107.27 & 551150 & 1429.66 & -2.48 \\
\hline & 0.4 & & & & 551554 & 1150.46 & 546455 & 1283.46 & -0.92 \\
\hline P28 & 0.5 & 200 & 10 & 150 & 550006 & 845.09 & 546211 & 901.12 & -0.69 \\
\hline & 0.6 & & & & 548151 & 679.59 & 543161 & 850.63 & -0.91 \\
\hline & 0.7 & & & & 546181 & 835.08 & 539990 & 965.50 & -1.13 \\
\hline & 0.8 & & & & 545049 & 869.30 & 538293 & 1444.22 & -1.24 \\
\hline & 0.9 & & & & 543203 & 937.76 & 522946 & 1065.27 & -3.73 \\
\hline & 0.1 & & & & 621423 & 1031.98 & 619749 & 810.58 & -0.27 \\
\hline & 0.2 & & & & 624921 & 755.35 & 618833 & 817.09 & -0.97 \\
\hline & 0.3 & & & & 620040 & 711.57 & 617782 & 991.29 & -0.36 \\
\hline & 0.4 & & & & 617601 & 867.88 & 615738 & 958.45 & -0.30 \\
\hline P29 & 0.5 & 200 & 10 & 70 & 623408 & 1135.24 & 614301 & 1168.53 & -1.46 \\
\hline & 0.6 & & & & 614480 & 799.56 & 612048 & 1337.13 & -0.40 \\
\hline & 0.7 & & & & 612960 & 1095.73 & 611365 & 948.58 & -0.26 \\
\hline & 0.8 & & & & 611261 & 1126.74 & 609791 & 969.75 & -0.24 \\
\hline & 0.9 & & & & 609581 & 846.60 & 608927 & 712.33 & -0.11 \\
\hline & 0.1 & & & & 522946 & 935.56 & 520709 & 1214.20 & -0.43 \\
\hline & 0.2 & & & & 521952 & 696.92 & 518710 & 871.10 & -0.62 \\
\hline & 0.3 & & & & 520505 & 730.31 & 516812 & 1182.22 & -0.71 \\
\hline & 0.4 & & & & 519262 & 780.89 & 516352 & 1289.81 & -0.56 \\
\hline P30 & 0.5 & 200 & 10 & 150 & 517999 & 707.29 & 516019 & 1335.97 & -0.38 \\
\hline & 0.6 & & & & 516930 & 1052.88 & 515388 & 1212.71 & -0.30 \\
\hline & 0.7 & & & & 515604 & 954.61 & 513938 & 1054.47 & -0.32 \\
\hline & 0.8 & & & & 514221 & 687.26 & 511705 & 1073.18 & -0.49 \\
\hline & 0.9 & & & & 497335 & 1020.75 & 492080 & 1113.48 & -1.06 \\
\hline
\end{tabular}


TABLE 4: Results of different rate between the cost of recovered products and the cost of new products.

\begin{tabular}{|c|c|c|c|c|c|c|c|c|c|}
\hline \multirow{2}{*}{ Prob. ID } & \multirow{2}{*}{$c^{m}: c^{r}$} & \multirow{2}{*}{$|I|$} & \multirow{2}{*}{$|J|$} & \multirow{2}{*}{$Q$} & \multicolumn{2}{|c|}{ TS } & \multicolumn{2}{|c|}{ NTS } & \multirow{2}{*}{ Gap $1(\%)$} \\
\hline & & & & & UB & Time & UB & Time & \\
\hline \multirow{5}{*}{ P1 } & 1 & \multirow{5}{*}{20} & \multirow{5}{*}{5} & \multirow{5}{*}{70} & 68142 & 3.00 & 68142 & 2.78 & 0.00 \\
\hline & 1.25 & & & & 67402 & 2.68 & 67402 & 2.39 & 0.00 \\
\hline & 1.5 & & & & 67211 & 2.14 & 66964 & 2.61 & -0.37 \\
\hline & 1.75 & & & & 66898 & 3.15 & 66649 & 2.54 & -0.37 \\
\hline & 2 & & & & 66496 & 2.76 & 66414 & 2.79 & -0.12 \\
\hline \multirow{5}{*}{ P2 } & 1 & \multirow{5}{*}{20} & \multirow{5}{*}{5} & \multirow{5}{*}{150} & 43008 & 3.33 & 43008 & 2.35 & 0.00 \\
\hline & 1.25 & & & & 42964 & 3.11 & 42964 & 2.54 & 0.00 \\
\hline & 1.5 & & & & 42934 & 2.49 & 42934 & 2.22 & 0.00 \\
\hline & 1.75 & & & & 42913 & 2.40 & 42913 & 3.15 & 0.00 \\
\hline & 2 & & & & 42897 & 3.55 & 42897 & 3.19 & 0.00 \\
\hline \multirow{5}{*}{ P3 } & 1 & \multirow{5}{*}{20} & & & 61680 & 2.72 & 61680 & 2.82 & 0.00 \\
\hline & 1.25 & & & & 61028 & 2.46 & 61028 & 2.81 & 0.00 \\
\hline & 1.5 & & 5 & 70 & 60595 & 2.47 & 60595 & 2.54 & 0.00 \\
\hline & 1.75 & & & & 60367 & 2.59 & 60285 & 2.71 & -0.14 \\
\hline & 2 & & & & 60132 & 2.52 & 60052 & 2.50 & -0.13 \\
\hline & 1 & & & & 40458 & 2.45 & 40458 & 2.05 & 0.00 \\
\hline & 1.25 & & & & 40391 & 2.18 & 40391 & 2.36 & 0.00 \\
\hline $\mathrm{P} 4$ & 1.5 & 20 & 5 & 150 & 40346 & 2.86 & 40346 & 2.96 & 0.00 \\
\hline & 1.75 & & & & 40314 & 2.69 & 40314 & 2.32 & 0.00 \\
\hline & 2 & & & & 40290 & 1.92 & 40290 & 2.28 & 0.00 \\
\hline & 1 & & & & 117832 & 26.39 & 116378 & 25.98 & -1.23 \\
\hline & 1.25 & & & & 117029 & 25.68 & 114692 & 26.63 & -2.00 \\
\hline P5 & 1.5 & 50 & 5 & 70 & 115617 & 26.14 & 114374 & 25.88 & -1.08 \\
\hline & 1.75 & & & & 115126 & 26.70 & 114022 & 26.30 & -0.96 \\
\hline & 2 & & & & 113413 & 25.84 & 112590 & 25.75 & -0.73 \\
\hline & 1 & & & & 90053 & 22.94 & 87484 & 20.19 & -2.85 \\
\hline & 1.25 & & & & 88307 & 24.22 & 86991 & 20.15 & -1.49 \\
\hline P6 & 1.5 & 50 & 5 & 150 & 87796 & 23.82 & 86910 & 23.21 & -1.01 \\
\hline & 1.75 & & & & 86976 & 20.81 & 86535 & 22.24 & -0.51 \\
\hline & 2 & & & & 86703 & 22.88 & 86394 & 17.23 & -0.36 \\
\hline & 1 & & & & 123939 & 18.23 & 122295 & 21.88 & -1.33 \\
\hline & 1.25 & & & & 121637 & 19.25 & 120958 & 16.91 & -0.56 \\
\hline P7 & 1.5 & 50 & 5 & 70 & 120474 & 18.75 & 119532 & 20.84 & -0.78 \\
\hline & 1.75 & & & & 119790 & 21.88 & 118308 & 21.61 & -1.24 \\
\hline & 2 & & & & 118183 & 16.95 & 117858 & 21.77 & -0.27 \\
\hline & 1 & & & & 100524 & 17.41 & 100439 & 25.51 & -0.08 \\
\hline & 1.25 & & & & 99371 & 28.59 & 99067 & 29.73 & -0.31 \\
\hline P8 & 1.5 & 50 & 5 & 150 & 98231 & 24.37 & 97807 & 29.53 & -0.43 \\
\hline & 1.75 & & & & 97514 & 24.48 & 96608 & 22.80 & -0.93 \\
\hline & 2 & & & & 96942 & 25.15 & 96560 & 17.95 & -0.39 \\
\hline & 1 & & & & 117880 & 27.00 & 115956 & 22.17 & -1.63 \\
\hline & 1.25 & & & & 116053 & 30.39 & 114876 & 35.22 & -1.01 \\
\hline P9 & 1.5 & 50 & 5 & 70 & 114690 & 24.03 & 113420 & 28.06 & -1.11 \\
\hline & 1.75 & & & & 113128 & 19.20 & 112769 & 28.95 & -0.32 \\
\hline & 2 & & & & 112006 & 28.62 & 111756 & 26.96 & -0.22 \\
\hline & 1 & & & & 88057 & 23.26 & 87883 & 31.21 & -0.20 \\
\hline & 1.25 & & & & 86485 & 25.83 & 86222 & 31.48 & -0.30 \\
\hline P10 & 1.5 & 50 & 5 & 150 & 85367 & 31.62 & 84589 & 19.58 & -0.91 \\
\hline & 1.75 & & & & 83386 & 26.10 & 83478 & 22.37 & 0.11 \\
\hline & 2 & & & & 83455 & 25.45 & 83235 & 21.90 & -0.26 \\
\hline
\end{tabular}


TABLE 4: Continued.

\begin{tabular}{|c|c|c|c|c|c|c|c|c|c|}
\hline \multirow{2}{*}{ Prob. ID } & \multirow{2}{*}{$c^{m}: c^{r}$} & \multirow{2}{*}{$|I|$} & \multirow{2}{*}{$|J|$} & \multirow{2}{*}{$Q$} & \multicolumn{2}{|c|}{ TS } & \multicolumn{2}{|c|}{ NTS } & \multirow{2}{*}{ Gap $1(\%)$} \\
\hline & & & & & UB & Time & UB & Time & \\
\hline \multirow{5}{*}{ P11 } & 1 & \multirow{5}{*}{50} & \multirow{5}{*}{5} & \multirow{5}{*}{70} & 114793 & 20.04 & 112544 & 25.68 & -1.96 \\
\hline & 1.25 & & & & 112136 & 19.99 & 111395 & 27.60 & -0.66 \\
\hline & 1.5 & & & & 111087 & 20.16 & 111062 & 29.96 & -0.02 \\
\hline & 1.75 & & & & 110640 & 27.13 & 109472 & 22.12 & -1.06 \\
\hline & 2 & & & & 110405 & 22.60 & 108934 & 26.43 & -1.33 \\
\hline \multirow{5}{*}{ P12 } & 1 & \multirow{5}{*}{50} & \multirow{5}{*}{5} & \multirow{5}{*}{150} & 87232 & 24.63 & 86433 & 29.55 & -0.04 \\
\hline & 1.25 & & & & 86410 & 22.09 & 86199 & 18.89 & -0.57 \\
\hline & 1.5 & & & & 85173 & 27.98 & 83868 & 19.14 & -1.94 \\
\hline & 1.75 & & & & 84523 & 21.53 & 83656 & 15.63 & -2.18 \\
\hline & 2 & & & & 83027 & 18.38 & 82912 & 20.22 & -1.46 \\
\hline \multirow{5}{*}{$\mathrm{P} 13$} & 1 & \multirow{5}{*}{100} & & & 364895 & 155.68 & 361755 & 159.32 & -0.86 \\
\hline & 1.25 & & & & 363309 & 160.69 & 360230 & 160.93 & -0.85 \\
\hline & 1.5 & & 5 & 70 & 362096 & 163.84 & 359999 & 168.78 & -0.58 \\
\hline & 1.75 & & & & 359974 & 157.96 & 356857 & 141.78 & -0.87 \\
\hline & 2 & & & & 355624 & 156.57 & 339404 & 161.89 & -4.56 \\
\hline & 1 & & & & 305053 & 164.11 & 300033 & 139.62 & -1.65 \\
\hline & 1.25 & & & & 303135 & 141.89 & 296364 & 121.95 & -2.23 \\
\hline P14 & 1.5 & 100 & 5 & 150 & 301834 & 139.24 & 295118 & 121.68 & -2.23 \\
\hline & 1.75 & & & & 298777 & 139.76 & 294442 & 133.88 & -1.45 \\
\hline & 2 & & & & 291740 & 133.99 & 276706 & 135.66 & -5.15 \\
\hline & 1 & & & & 296619 & 124.99 & 258199 & 125.21 & -12.95 \\
\hline & 1.25 & & & & 260317 & 137.47 & 254461 & 128.82 & -2.25 \\
\hline P15 & 1.5 & 100 & 5 & 70 & 249922 & 115.05 & 247152 & 183.34 & -1.11 \\
\hline & 1.75 & & & & 249195 & 132.22 & 244535 & 154.43 & -1.87 \\
\hline & 2 & & & & 247529 & 149.44 & 241351 & 171.13 & -2.50 \\
\hline & 1 & & & & 233346 & 120.31 & 223398 & 126.11 & -4.26 \\
\hline & 1.25 & & & & 229687 & 129.75 & 220760 & 114.91 & -3.89 \\
\hline P16 & 1.5 & 100 & 5 & 150 & 226932 & 127.63 & 218861 & 117.39 & -3.56 \\
\hline & 1.75 & & & & 216624 & 137.42 & 214174 & 115.09 & -1.13 \\
\hline & 2 & & & & 211223 & 128.79 & 205238 & 122.39 & -2.83 \\
\hline & 1 & & & & 267713 & 134.93 & 265309 & 110.60 & -0.90 \\
\hline & 1.25 & & & & 266067 & 132.17 & 262547 & 122.34 & -1.32 \\
\hline P17 & 1.5 & 100 & 5 & 70 & 263994 & 130.92 & 260267 & 110.49 & -1.41 \\
\hline & 1.75 & & & & 261711 & 116.78 & 259735 & 150.61 & -0.76 \\
\hline & 2 & & & & 260804 & 170.99 & 252673 & 124.40 & -3.12 \\
\hline & 1 & & & & 226178 & 124.86 & 224348 & 139.70 & -0.81 \\
\hline & 1.25 & & & & 223053 & 127.08 & 219578 & 134.79 & -1.56 \\
\hline P18 & 1.5 & 100 & 5 & 150 & 220970 & 115.28 & 218823 & 184.85 & -0.97 \\
\hline & 1.75 & & & & 219154 & 158.19 & 216341 & 129.00 & -1.28 \\
\hline & 2 & & & & 217751 & 159.37 & 209257 & 182.73 & -3.90 \\
\hline & 1 & & & & 412240 & 135.46 & 409643 & 141.11 & -0.63 \\
\hline & 1.25 & & & & 406518 & 139.13 & 403969 & 173.98 & -0.63 \\
\hline P19 & 1.5 & 100 & 10 & 70 & 404199 & 137.06 & 401006 & 167.12 & -0.79 \\
\hline & 1.75 & & & & 403475 & 136.64 & 400046 & 178.88 & -0.85 \\
\hline & 2 & & & & 401608 & 136.54 & 394231 & 149.81 & -1.84 \\
\hline & 1 & & & & 366576 & 105.10 & 364188 & 166.50 & -0.65 \\
\hline & 1.25 & & & & 364342 & 123.15 & 362564 & 100.25 & -0.49 \\
\hline P20 & 1.5 & 100 & 10 & 150 & 360397 & 153.27 & 358732 & 143.08 & -0.46 \\
\hline & 1.75 & & & & 357502 & 158.04 & 356178 & 114.59 & -0.37 \\
\hline & 2 & & & & 350419 & 100.43 & 345948 & 154.21 & -1.28 \\
\hline
\end{tabular}


TABle 4: Continued.

\begin{tabular}{|c|c|c|c|c|c|c|c|c|c|}
\hline \multirow{2}{*}{ Prob. ID } & \multirow{2}{*}{$c^{m}: c^{r}$} & \multirow{2}{*}{$|I|$} & \multirow{2}{*}{$|J|$} & \multirow{2}{*}{$Q$} & \multicolumn{2}{|c|}{ TS } & \multicolumn{2}{|c|}{ NTS } & \multirow{2}{*}{ Gap 1 (\% } \\
\hline & & & & & UB & Time & UB & Time & \\
\hline \multirow{5}{*}{$\mathrm{P} 21$} & 1 & \multirow{5}{*}{100} & \multirow{5}{*}{10} & \multirow{5}{*}{70} & 337749 & 113.31 & 334240 & 100.11 & -1.04 \\
\hline & 1.25 & & & & 333248 & 114.45 & 330768 & 112.04 & -0.74 \\
\hline & 1.5 & & & & 330981 & 127.66 & 329900 & 133.01 & -0.33 \\
\hline & 1.75 & & & & 329982 & 143.46 & 325218 & 139.32 & -1.44 \\
\hline & 2 & & & & 327911 & 189.02 & 322382 & 150.34 & -1.69 \\
\hline \multirow{5}{*}{ P22 } & 1 & \multirow{5}{*}{100} & \multirow{5}{*}{10} & \multirow{5}{*}{150} & 300245 & 112.22 & 297406 & 94.24 & -0.95 \\
\hline & 1.25 & & & & 293642 & 127.66 & 291212 & 119.95 & -0.83 \\
\hline & 1.5 & & & & 290938 & 186.05 & 287471 & 192.95 & -1.19 \\
\hline & 1.75 & & & & 288716 & 183.72 & 285885 & 127.07 & -0.98 \\
\hline & 2 & & & & 286473 & 132.29 & 281933 & 125.07 & -1.58 \\
\hline \multirow{5}{*}{$\mathrm{P} 23$} & 1 & \multirow{5}{*}{100} & & & 369343 & 127.38 & 364871 & 132.22 & -1.21 \\
\hline & 1.25 & & & & 364871 & 152.70 & 359677 & 141.96 & -1.42 \\
\hline & 1.5 & & 10 & 70 & 359613 & 147.70 & 355286 & 152.78 & -1.20 \\
\hline & 1.75 & & & & 358094 & 155.05 & 354980 & 142.97 & -0.87 \\
\hline & 2 & & & & 341610 & 146.96 & 320343 & 172.26 & -6.23 \\
\hline & 1 & & & & 328663 & 124.91 & 299018 & 132.71 & -9.02 \\
\hline & 1.25 & & & & 325042 & 125.13 & 295553 & 137.29 & -9.07 \\
\hline P24 & 1.5 & 100 & 10 & 150 & 323847 & 156.83 & 291860 & 135.43 & -9.88 \\
\hline & 1.75 & & & & 299214 & 135.60 & 288433 & 157.16 & -3.60 \\
\hline & 2 & & & & 294706 & 121.22 & 281938 & 168.67 & -4.33 \\
\hline & 1 & & & & 665281 & 829.11 & 653633 & 1026.07 & -1.75 \\
\hline & 1.25 & & & & 657935 & 834.27 & 652114 & 1061.26 & -0.88 \\
\hline P25 & 1.5 & 200 & 10 & 70 & 655818 & 823.79 & 651574 & 984.23 & -0.65 \\
\hline & 1.75 & & & & 649604 & 820.10 & 646761 & 1055.81 & -0.44 \\
\hline & 2 & & & & 642489 & 820.87 & 641864 & 1139.61 & -0.10 \\
\hline & 1 & & & & 581623 & 611.52 & 580270 & 618.26 & -0.23 \\
\hline & 1.25 & & & & 579961 & 608.25 & 569283 & 626.20 & -1.84 \\
\hline P26 & 1.5 & 200 & 10 & 150 & 574684 & 604.60 & 536008 & 613.79 & -6.73 \\
\hline & 1.75 & & & & 556955 & 621.66 & 535570 & 623.55 & -3.84 \\
\hline & 2 & & & & 554052 & 621.92 & 528999 & 622.53 & -4.52 \\
\hline & 1 & & & & 773781 & 793.09 & 662523 & 778.08 & -14.38 \\
\hline & 1.25 & & & & 713012 & 819.64 & 655573 & 803.25 & -8.06 \\
\hline P27 & 1.5 & 200 & 10 & 70 & 681260 & 804.27 & 642081 & 739.51 & -5.75 \\
\hline & 1.75 & & & & 635834 & 770.17 & 632602 & 723.11 & -0.51 \\
\hline & 2 & & & & 632061 & 739.14 & 623493 & 704.85 & -1.36 \\
\hline & 1 & & & & 576983 & 666.69 & 555923 & 987.24 & -3.65 \\
\hline & 1.25 & & & & 568736 & 675.82 & 546433 & 859.83 & -3.92 \\
\hline P28 & 1.5 & 200 & 10 & 150 & 564064 & 674.70 & 543330 & 859.83 & -3.68 \\
\hline & 1.75 & & & & 560726 & 914.60 & 535818 & 761.02 & -4.44 \\
\hline & 2 & & & & 534830 & 977.03 & 522356 & 795.76 & -2.33 \\
\hline & 1 & & & & 666957 & 790.82 & 636582 & 820.80 & -4.55 \\
\hline & 1.25 & & & & 658832 & 832.47 & 633813 & 1119.70 & -3.80 \\
\hline P29 & 1.5 & 200 & 10 & 70 & 653454 & 1149.63 & 621988 & 965.82 & -4.82 \\
\hline & 1.75 & & & & 622078 & 801.89 & 619907 & 825.17 & -0.35 \\
\hline & 2 & & & & 616051 & 863.47 & 608644 & 1080.37 & -1.20 \\
\hline & 1 & & & & 542071 & 734.01 & 540384 & 705.36 & -0.31 \\
\hline & 1.25 & & & & 525159 & 727.55 & 524172 & 786.56 & -0.19 \\
\hline P30 & 1.5 & 200 & 10 & 150 & 521758 & 716.37 & 510933 & 763.03 & -2.07 \\
\hline & 1.75 & & & & 518474 & 787.04 & 506479 & 752.31 & -2.31 \\
\hline & 2 & & & & 515142 & 755.66 & 502884 & 698.85 & -2.38 \\
\hline
\end{tabular}




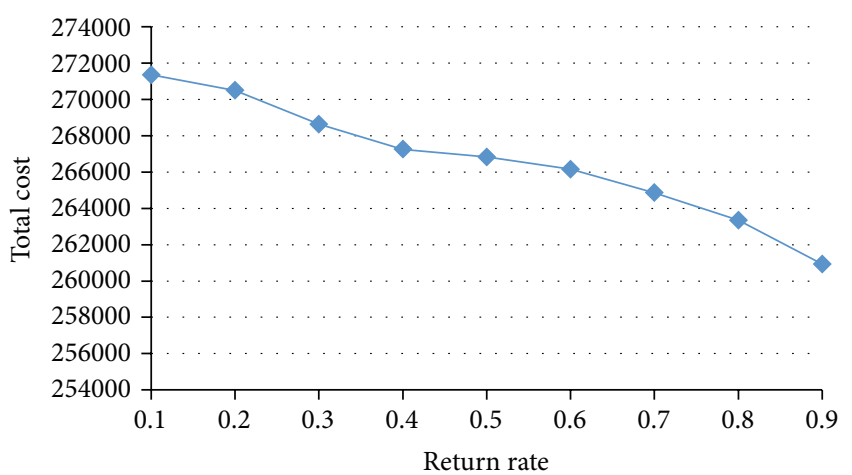

FIgURE 3: Results of different return rates.

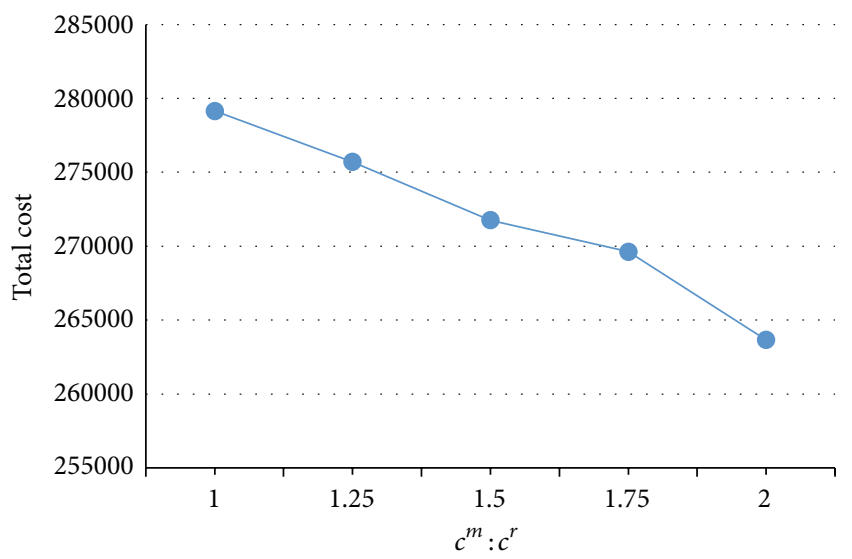

Figure 4: Results of different rate between the cost of recovered products and the cost of new products.

and randomly generated instances of LIRP-FRL with various problem sizes demonstrate the effectiveness and the efficiency of the new tabu search, which can find high quality solution with a reasonable time. Sensitivity analyses are also conducted to investigate the intrinsic management insights of the problems.

For future research, to be more close to the reality, the stochastic demand of customer should also be considered in the LIRP-FRL.

\section{Competing Interests}

The authors declare that they have no competing interests.

\section{Acknowledgments}

The research presented in this paper is supported by the National Natural Science Foundation Project of China (71390331, 71572138, 71271168, and 71371150), the National Social Science Foundation Project of China (12\&ZD070), the National Soft Science Project of China (2014GXS4D151), the Soft Science Project of Shaanxi Province (2014KRZ04), the Program for New Century Excellent Talents in University (NCET-13-0460), and the Fundamental Research Funds for the Central Universities.

\section{References}

[1] A. A. Javid and N. Azad, "Incorporating location, routing and inventory decisions in supply chain network design," Transportation Research, Part E: Logistics and Transportation Review, vol. 46, no. 5, pp. 582-597, 2010.

[2] S. C. Liu and S. B. Lee, "A two-phase heuristic method for the multi-depot location routing problem taking inventory control decisions into consideration," International Journal of Advanced Manufacturing Technology, vol. 22, no. 11-12, pp. 941-950, 2003.

[3] S. Viswanathan and K. Mathur, "Integrating routing and inventory decisions in one-warehouse multiretailer multiproduct distribution systems," Management Science, vol. 43, no. 3, pp. 294-312, 1997.

[4] Y. Zhang, M. Qi, L. Miao, and E. Liu, "Hybrid metaheuristic solutions to inventory location routing problem," Transportation Research Part E: Logistics and Transportation Review, vol. 70, no. 1, pp. 305-323, 2014.

[5] D. Ambrosino and M. G. Scutella, "Distribution network design: new problems and related models," European Journal of Operational Research, vol. 165, no. 3, pp. 610-624, 2005.

[6] Z.-J. M. Shen and L. Qi, "Incorporating inventory and routing costs in strategic location models," European Journal of Operational Research, vol. 179, no. 2, pp. 372-389, 2007.

[7] W. J. Guerrero, C. Prodhon, N. Velasco, and C. A. Amaya, "A relax-and-price heuristic for the inventory-location-routing problem," International Transactions in Operational Research, vol. 22, no. 1, pp. 129-148, 2015.

[8] V. Ravi, R. Shankar, and M. K. Tiwari, "Analyzing alternatives in reverse logistics for end-of-life computers: ANP and balanced scorecard approach," Computers \& Industrial Engineering, vol. 48, no. 2, pp. 327-356, 2005.

[9] B. Vahdani and M. Naderi-Beni, "A mathematical programming model for recycling network design under uncertainty: an interval-stochastic robust optimization model," The International Journal of Advanced Manufacturing Technology, vol. 73, no. 5-8, pp. 1057-1071, 2014.

[10] C.-T. Chen, P.-F. Pai, and W.-Z. Hung, "An integrated methodology using linguistic PROMETHEE and maximum deviation method for third-party logistics supplier selection," International Journal of Computational Intelligence Systems, vol. 3, no. 4, pp. 438-451, 2010.

[11] B. Vahdani, J. Razmi, and R. Tavakkoli-Moghaddam, "Fuzzy possibilistic modeling for closed loop recycling collection networks," Environmental Modeling \& Assessment, vol. 17, no. 6, pp. 623-637, 2012.

[12] B. Vahdani, R. Tavakkoli-Moghaddam, and F. Jolai, "Reliable design of a logistics network under uncertainty: a fuzzy possibilistic-queuing model," Applied Mathematical Modelling, vol. 37, no. 5, pp. 3254-3268, 2013.

[13] B. Vahdani and M. Mohammadi, "A bi-objective intervalstochastic robust optimization model for designing closed loop supply chain network with multi-priority queuing system," International Journal of Production Economics, vol. 170, pp. 6787, 2015.

[14] K. Richter, "The EOQ repair and waste disposal model with variable setup numbers," European Journal of Operational Research, vol. 95, no. 2, pp. 313-324, 1996.

[15] J. Shi, G. Zhang, and J. Sha, "Optimal production planning for a multi-product closed loop system with uncertain demand and return," Computers \& Operations Research, vol. 38, no. 3, pp. 641-650, 2011. 
[16] E. E. Zachariadis and C. T. Kiranoudis, "A local search metaheuristic algorithm for the vehicle routing problem with simultaneous pick-ups and deliveries," Expert Systems with Applications, vol. 38, no. 3, pp. 2717-2726, 2011.

[17] F. P. Goksal, I. Karaoglan, and F. Altiparmak, "A hybrid discrete particle swarm optimization for vehicle routing problem with simultaneous pickup and delivery," Computers \& Industrial Engineering, vol. 65, no. 1, pp. 39-53, 2013.

[18] H. Liu, Q. Zhang, and W. Wang, "Research on location-routing problem of reverse logistics with grey recycling demands based on PSO," Grey Systems: Theory and Application, vol. 1, no. 1, pp. 97-104, 2011.

[19] V. F. Yu and S.-W. Lin, "Multi-start simulated annealing heuristic for the location routing problem with simultaneous pickup and delivery," Applied Soft Computing, vol. 24, pp. 284-290, 2014.

[20] Z. Wang, D.-Q. Yao, and P. Huang, "A new location-inventory policy with reverse logistics applied to B2C e-markets of China," International Journal of Production Economics, vol. 107, no. 2, pp. 350-363, 2007.

[21] Y. Li, M. Lu, and B. Liu, "A two-stage algorithm for the closedloop location-inventory problem model considering returns in E-commerce," Mathematical Problems in Engineering, vol. 2014, Article ID 260869, 9 pages, 2014.

[22] Y. Li, H. Guo, L. Wang, and J. Fu, "A hybrid genetic-simulated annealing algorithm for the location-inventory- routing problem considering returns under E-supply chain environment," The Scientific World Journal, vol. 2013, Article ID 125893, 10 pages, 2013.

[23] D. P. Adler, V. Kumar, P. A. Ludewig, and J. W. Sutherland, "Comparing energy and other measures of environmental performance in the original manufacturing and remanufacturing of engine components," in Proceedings of the ASME International Conference on Manufacturing Science and Engineering, pp. 851-860, October 2007.

[24] J.-M. Belenguer, E. Benavent, C. Prins, C. Prodhon, and R. Wolfler Calvo, "A branch-and-cut method for the capacitated location-routing problem," Computers \& Operations Research, vol. 38, no. 6, pp. 931-941, 2011.

[25] R. H. Teunter, "Economic ordering quantities for recoverable item inventory systems," Naval Research Logistics, vol. 48, no. 6, pp. 484-495, 2001.

[26] F. Glover, "Future paths for integer programming and links to artificial intelligence," Computers \& Operations Research, vol. 13, no. 5, pp. 533-549, 1986.

[27] F. Glover, "Tabu search-part I," ORSA Journal on Computing, vol. 1, no. 3, pp. 190-206, 1989.

[28] D. Habet, "Tabu search to solve real-life combinatorial optimization problems: a case of study," Foundations of Computational Intelligence, vol. 3, pp. 129-151, 2009.

[29] V. F. Yu, S.-W. Lin, W. Lee, and C.-J. Ting, "A simulated annealing heuristic for the capacitated location routing problem," Computers and Industrial Engineering, vol. 58, no. 2, pp. 288299, 2010.

[30] D. J. Rosenkrantz, R. E. Stearns, and P. M. Lewis, "Approximate algorithms for the traveling salesperson problem," in Proceedings of the IEEE Conference Record of 15th Annual Symposium on Switching and Automata Theory, pp. 33-42, October 1974.

[31] C. Prins, C. Prodhon, and R. Wolfler-Calvo, "Nouveaux algorithmes pour le problème de localisation et routage sous contraintes de capacité," MOSIM, vol. 4, no. 2, pp. 1115-1122, 2004. 


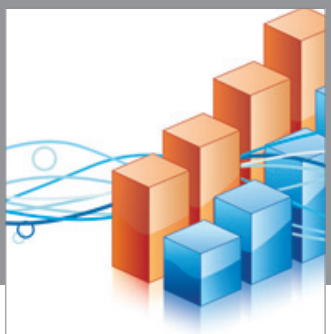

Advances in

Operations Research

vatem alat4

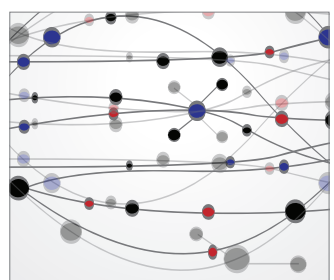

\section{The Scientific} World Journal
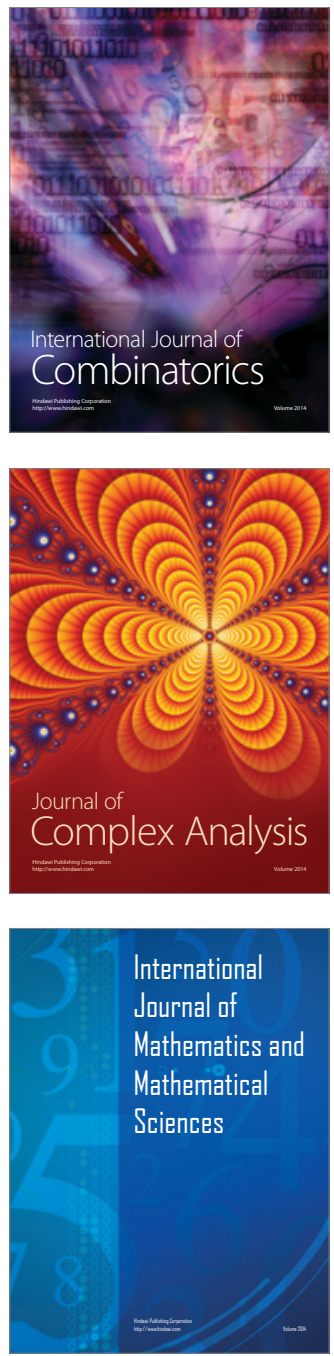
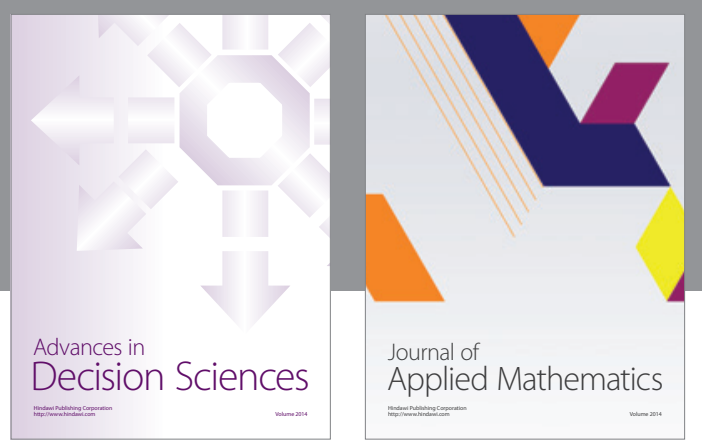

Algebra

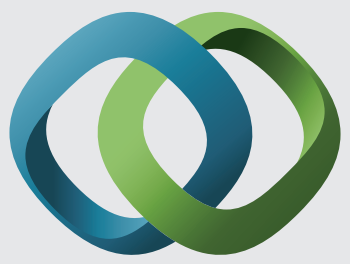

\section{Hindawi}

Submit your manuscripts at

http://www.hindawi.com
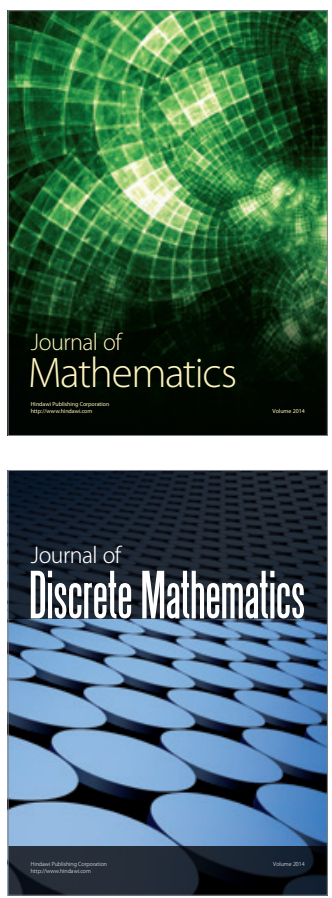

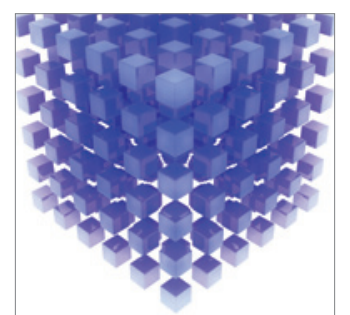

Mathematical Problems in Engineering
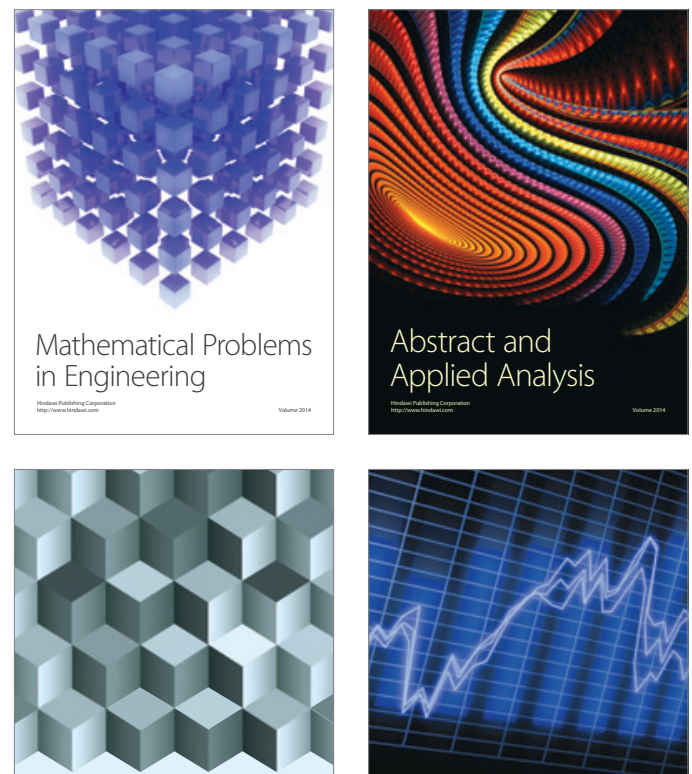

Journal of

Function Spaces

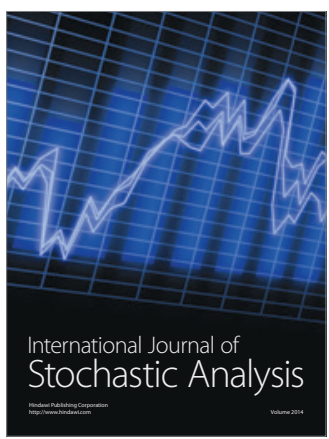

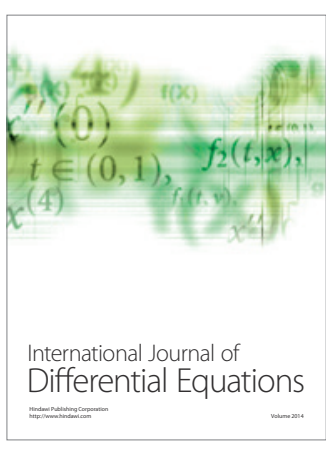
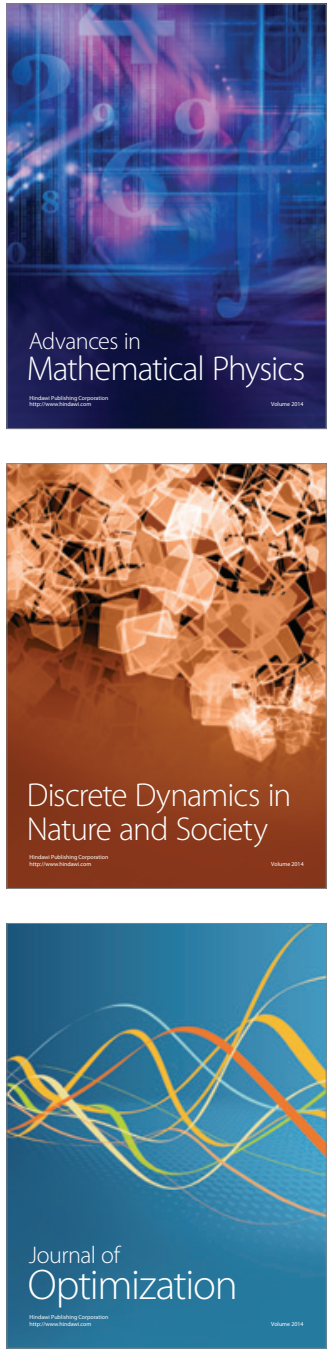